\title{
On the ill-posedness of the compressible Navier-Stokes equations in the critical Besov spaces
}

\author{
Qionglei Chen, Changxing Miao and Zhifei Zhang
}

\begin{abstract}
We prove the ill-posedness of the 3-D baratropic NavierStokes equation for the initial density and velocity belonging to the critical Besov space $\left(\dot{B}_{p, 1}^{3 / p}+\bar{\rho}, \dot{B}_{p, 1}^{3 / p-1}\right)$ for $p>6$ in the sense that a "norm inflation" happens in finite time, here $\bar{\rho}$ is a positive constant. While, the compressible viscous heat-conductive flows is ill-posed for the initial density, velocity and temperature belonging to the critical Besov space $\left(\dot{B}_{p, 1}^{3 / p}+\bar{\rho}, \dot{B}_{p, 1}^{3 / p-1}, \dot{B}_{p, 1}^{3 / p-2}\right)$ for $p>3$.
\end{abstract}

\section{Introduction}

The full compressible Navier-Stokes equations read as follows:

$$
\left\{\begin{array}{l}
\partial_{t} \rho+\operatorname{div}(\rho u)=0, \\
\partial_{t}(\rho u)+\operatorname{div}(\rho u \otimes u)=\operatorname{div} \tau, \\
\partial_{t}\left(\rho u\left(e+\frac{|u|^{2}}{2}\right)\right)+\operatorname{div}\left(\rho u\left(e+\frac{|u|^{2}}{2}\right)\right)=\operatorname{div}(\tau \cdot u+\kappa \nabla \theta),
\end{array}\right.
$$

where $\rho(t, x), u(t, x), e(t, x)$ denote the density, velocity of the fluid and the internal energy per unit mass respectively, $\kappa>0$ is the thermal conduction parameter, and $\theta$ is the temperature. The internal stress tensor $\tau$ is given by

$$
\tau=2 \nu \mathrm{D}(u)+(\lambda \operatorname{div} u-p) I,
$$

where $\mathrm{D}(u)=\frac{1}{2}\left(\nabla u+\nabla u^{\top}\right)$, the constants $\mu, \lambda$ are the viscosity coefficients satisfying

$$
\mu>0 \text { and } \lambda+2 \mu>0 \text {. }
$$

Mathematics Subject Classification (2010): Primary 35B35; Secondary 35Q30. Keywords: Compressible Navier-Stokes equations, ill-posedness, Besov space. 
For the ideal gas, $e=c_{V} \theta, P=\rho R \theta$ for some constants $c_{V}>0, R>0$. In such case, the system (1.1) can be rewritten as

$$
\left\{\begin{array}{l}
\partial_{t} \rho+\operatorname{div}(\rho u)=0 \\
\partial_{t}(\rho u)+\operatorname{div}(\rho u \otimes u)-\mu \Delta u-(\lambda+\mu) \nabla \operatorname{div} u+\nabla P=0 \\
c_{V}\left(\partial_{t}(\rho \theta)+\operatorname{div}(\rho u \theta)\right)-\kappa \Delta \theta+P \operatorname{div} u=\frac{\mu}{2}\left|\nabla u+(\nabla u)^{\top}\right|^{2}+\lambda|\operatorname{div} u|^{2}
\end{array}\right.
$$

Here we denote by $|A|^{2}$ the trace of the matrix $A A^{\top}$.

When the pressure depends only on the density, we get the baratropic NavierStokes equations

$$
\left\{\begin{array}{l}
\partial_{t} \rho+\operatorname{div}(\rho u)=0 \\
\partial_{t}(\rho u)+\operatorname{div}(\rho u \otimes u)-\mu \Delta u-(\lambda+\mu) \nabla \operatorname{div} u+\nabla P=0
\end{array}\right.
$$

In this paper, we are concerned with the Cauchy problem of the system (1.2) and (1.3) in $\mathbb{R}^{+} \times \mathbb{R}^{3}$ together with the initial data

$$
\left.(\rho, u, \theta)\right|_{t=0}=\left(\rho_{0}, u_{0}, \theta_{0}\right), \quad \text { and }\left.\quad(\rho, u)\right|_{t=0}=\left(\rho_{0}, u_{0}\right),
$$

respectively.

The local existence and uniqueness of smooth solutions for the system (1.2) were proved by Nash [15] for smooth initial data without vacuum. MatsumuraNishida [14] proved that the solution is global in time for the data close to equilibrium. In the general case, the question of whether smooth solutions blow up in finite time is widely open, even in two dimensional case. For the initial density with compact support, Xin [18] proved that any non-zero smooth solutions of (1.2) will blow up in finite time. Recently, Sun-Wang-Zhang [16], [17] showed that smooth solution will not blow up as long as the upper bound of the density( and temperature for (1.2)) is bounded. We refer to the seminal books [13], [10] and references therein for the global existence of weak solutions.

Motivated by Fujita-Kato's theory on the incompressible Navier-Stokes equations [11], Danchin applied Fourier analysis method to study the well-posedness for the compressible Navier-Stokes equations in critical Besov spaces. Let us make it precise. It is easy to check that if $(\rho, u, \theta)$ is a solution of $(1.2)$, then

$$
\left(\rho_{\lambda}(t, x), u_{\lambda}(t, x), \theta_{\lambda}(t, x)\right) \stackrel{\text { def }}{=}\left(\rho\left(\lambda^{2} t, \lambda x\right), \lambda u\left(\lambda^{2} t, \lambda x\right), \lambda^{2} \theta\left(\lambda^{2} t, \lambda x\right)\right), \quad \lambda>0
$$

is also a solution of (1.2) provided the pressure law has been changed into $\lambda^{2} P$. A functional space is called critical if the associated norm is invariant under the transformation $(\rho, u, \theta) \rightarrow\left(\rho_{\lambda}, u_{\lambda}, \theta_{\lambda}\right)$ (up to a constant independent of $\lambda$ ). Roughly speaking, the system (1.2) is locally well-posed for the initial data

$$
\left(\rho_{0}-\bar{\rho}, u_{0}, \theta_{0}\right) \in \dot{B}_{p, 1}^{3 / p} \times\left(\dot{B}_{p, 1}^{3 / p-1}\right)^{3} \times \dot{B}_{p, 1}^{3 / p-2} \quad \text { with } p<3 ;
$$

and the system (1.3) is locally well-posed for the initial data

$$
\left(\rho_{0}-\bar{\rho}, u_{0}\right) \in \dot{B}_{p, 1}^{3 / p} \times\left(\dot{B}_{p, 1}^{3 / p-1}\right)^{3} \quad \text { with } p<6 .
$$


Here $\dot{B}_{p, q}^{s}$ is the homogeneous Besov space(see Definition 2.1). Moreover, the system (1.2) and (1.3) are globally well-posed if the initial data is small in the critical Besov space with $p=2$. We refer to [6], [7], [8], [9], [4] and references therein. Recently, Chen-Miao-Zhang and Charve-Danchin [5], [3] proved that the system (1.3) is globally well-posed for the small initial data in the hybrid critical Besov spaces, in which the part of high frequency of the initial data lies in the critical Besov spaces with $p>3$, and the part of low frequency lies in the critical Besov spaces with $p=2$. This result allows to generate a global solution for the highly oscillating initial velocity like $\sin \left(x_{1} / \varepsilon\right) \varphi(x)$, which is small in the hybrid critical Besov space.

A natural question is whether the system (1.2) and (1.3) are well-posed in the critical Besov spaces with $p \geq 3$ and $p \geq 6$ respectively. Recently, Bourgain and Pavlovic [2] and Germain [12] proved the ill-posedness of the incompressible Navier-Stokes equations in the largest critical space $\dot{B}_{\infty, \infty}^{-1}$. Motivated by the idea of [2], we prove that the system (1.3) is ill-posed in the critical Besov spaces with $p>6$.

Theorem 1.1. Let $\bar{\rho}$ be a positive constant and $p>6$. For any $\delta>0$, there exists initial data $\left(\rho_{0}, u_{0}\right)$ satisfying

$$
\left\|\rho_{0}-\bar{\rho}\right\|_{\dot{B}_{p, 1}^{3 / p}} \leq \delta, \quad\left\|u_{0}\right\|_{\dot{B}_{p, 1}^{3 / p-1}} \leq \delta
$$

such that a solution $(\rho, u)$ to the system (1.3) satisfies

$$
\|u(t)\|_{\dot{B}_{p, 1}^{3 / p-1}}>\frac{1}{\delta} \quad \text { for some } 0<t<\delta .
$$

While the system (1.2) is ill-posed in the critical Besov spaces with $p>3$.

Theorem 1.2. Let $\bar{\rho}$ be a positive constant and $p>3$. For any $\delta>0$, there exists initial data $\left(\rho_{0}, u_{0}, \theta_{0}\right)$ satisfying

$$
\left\|\rho_{0}-\bar{\rho}\right\|_{\dot{B}_{p, 1}^{3 / p}} \leq \delta, \quad\left\|u_{0}\right\|_{\dot{B}_{p, 1}^{3 / p-1}} \leq \delta, \quad\left\|\theta_{0}\right\|_{\dot{B}_{p, 1}^{3 / p-2}} \leq \delta
$$

such that a solution $(\rho, u, \theta)$ to the system (1.2) satisfies

$$
\|\theta(t)\|_{\dot{B}_{p, 1}^{3 / p-2}}>\frac{1}{\delta} \quad \text { for some } 0<t<\delta .
$$

Remark 1.3. In Theorem 1.1 and Theorem 1.2, the chosen initial data in fact belongs to $\mathcal{S}\left(\mathbb{R}^{3}\right)$. It is easy to prove (see [4], [9]) that the constructed solution $(\rho, u)$ of the system $(1.3)$ satisfies

$$
\rho-\bar{\rho} \in \widetilde{L}_{[0, T)}^{\infty} \dot{B}_{2,1}^{3 / 2}, \quad u \in \widetilde{L}_{[0, T)}^{\infty} \dot{B}_{2,1}^{1 / 2} \cap \widetilde{L}_{[0, T)}^{1} \dot{B}_{2,1}^{5 / 2}
$$

While, the solution $(\rho, u, \theta)$ of the system (1.2) satisfies

$\rho-\bar{\rho} \in \widetilde{L}_{[0, T)}^{\infty} \dot{B}_{2,1}^{3 / 2}, \quad u \in \widetilde{L}_{[0, T)}^{\infty} \dot{B}_{2,1}^{1 / 2} \cap \widetilde{L}_{[0, T)}^{1} \dot{B}_{2,1}^{5 / 2}, \quad \theta \in \widetilde{L}_{[0, T)}^{\infty} \dot{B}_{2,1}^{-1 / 2} \cap \widetilde{L}_{[0, T)}^{1} \dot{B}_{2,1}^{3 / 2}$.

For the space $\widetilde{L}_{[0, T)}^{\rho} \dot{B}_{r, s}^{\sigma}$, the reader can refer to Definition 2.2 in Section 2. 
Remark 1.4. Theorem 1.2 implies that it seems difficult to generate a global solution to the system (1.2) for the highly oscillating initial velocity as in [3], [5].

Remark 1.5. The question of whether the system (1.3) (or (1.2)) is well-posed in the critical Besov Space with $p=6$ (or $p=3$ ) is still open. Indeed, Lemma 3.1 fails for $p=6$ and Lemma 4.1 fails for $p=3$. However, these two basic lemmas assure that for the initial data of our construction the norm inflation happens in finite time for $p=6$ ( or $p=3$ ).

For the baratropic Navier-Stokes equations, the mechanism leading to the illposedness comes from the high-high frequency interaction into the low frequency of the nonlinear term $u_{1} \cdot \nabla u$, where $u_{1}=-(-\Delta)^{-1} \nabla \operatorname{div} u$ is the compressible part of $u$. Hence, the mechanism is different from the incompressible flow $(\operatorname{div} u=0)$. This is the main reason why the compressible flow is ill-posed in a smaller critical space. For the viscous heat-conductive flows, the mechanism leading to the illposedness comes from the high-high frequency interaction into the low frequency of the strong nonlinear terms $\left|\nabla u+(\nabla u)^{\top}\right|^{2}$ and $|\operatorname{div} u|^{2}$ in the temperature equation.

Following the idea of [2], we decompose the velocity $u$ into three parts,

$$
u(t)=U_{0}(t)+U_{1}(t)+U_{2}(t),
$$

where $U_{0}(t)=e^{t \Delta} u_{0}$ and $U_{1}(t)=\int_{0}^{t} e^{(t-\tau) \Delta} U_{0} \cdot \nabla U_{0}(\tau) d \tau$. The key step is to construct a suitable combination of plane waves for the initial velocity such that $\left\|U_{1}(t)\right\|_{\dot{B}_{p, 1}^{3 / p-1}}$ is big for some time $t>0$. Whilst, $\left\|U_{1}\right\|_{L_{t}^{1} \dot{B}_{q, 1}^{3 / q+1} \cap \widetilde{L}_{t}^{2} \dot{B}_{q, 1}^{3 / q}}$ is small for some $q<6$, which is important to ensure that the remainder part $\left\|U_{2}\right\|_{\widetilde{L}_{T}^{\infty} \dot{B}_{q, 1}^{3 / q-1} \cap L_{T}^{1} \dot{B}_{q, 1}^{3 / q+1}}$ is also small by the nonlinear estimates.

Acknowledgments. The authors are grateful to the referees for the invaluable comments and suggestions, which helped us improve the paper significantly.

\section{Some tools of Littlewood-Paley analysis}

\subsection{Littlewood-Paley decomposition and Besov sapces}

Choose a radial function $\varphi \in \mathcal{S}\left(\mathbb{R}^{3}\right)$ supported in $\mathcal{C}=\left\{\xi \in \mathbb{R}^{3}, 3 / 4 \leq|\xi| \leq 8 / 3\right\}$ such that

$$
\sum_{j \in \mathbb{Z}} \varphi\left(2^{-j} \xi\right)=1 \quad \text { for all } \xi \neq 0
$$

The frequency localization operator $\Delta_{j}$ and $S_{j}$ are defined by

$$
\Delta_{j} f=\varphi\left(2^{-j} D\right) f, \quad S_{j} f=\sum_{k \leq j-1} \Delta_{k} f \quad \text { for } j \in \mathbb{Z},
$$

respectively. The Fourier transform of $f$ is denoted by $\widehat{f}$ or $\mathcal{F} f$, and the inverse by $\mathcal{F}^{-1} f$. We denote by $\mathcal{S}_{h}{ }_{h}\left(\mathbb{R}^{3}\right)$ the set of the tempered distribution $f$ satisfying $\lim _{\lambda \rightarrow+\infty}\|\chi(\lambda D) f\|_{L^{\infty}}=0$ for some $\chi \in \mathcal{D}\left(\mathbb{R}^{3}\right)$ and $\chi(0)=0$. Let us introduce the homogeneous Besov space. 
Definition 2.1. Let $\sigma \in \mathbb{R}, 1 \leq r, s \leq+\infty$. The homogeneous Besov space $\dot{B}_{r, s}^{\sigma}$ is defined by

$$
\dot{B}_{r, s}^{\sigma} \stackrel{\text { def }}{=}\left\{f \in \mathcal{S}_{h}^{\prime}\left(\mathbb{R}^{3}\right):\|f\|_{\dot{B}_{r, s}^{\sigma}}<+\infty\right\}
$$

where

$$
\|f\|_{\dot{B}_{r, s}^{\sigma}}=\left\|\left\{2^{k \sigma}\left\|\Delta_{k} f\right\|_{L^{r}}\right\}_{j}\right\|_{\ell^{s}} .
$$

We next introduce the Chemin-Lerner type space $\widetilde{L}_{T}^{\rho} \dot{B}_{r, s}^{\sigma}$.

Definition 2.2. Let $\sigma \in \mathbb{R}, 1 \leq \rho, r, s \leq+\infty, 0<T \leq+\infty$. The space $\widetilde{L}_{T}^{\rho} \dot{B}_{r, s}^{\sigma}$ is defined as the set of all the distributions $f$ satisfying

$$
\|f\|_{\widetilde{L}_{T}^{\rho} \dot{B}_{r, s}^{\sigma}} \stackrel{\text { def }}{=}\left\|\left\{2^{k \sigma}\left\|\Delta_{k} f(t)\right\|_{L^{\rho}\left([0, T) ; L^{r}\left(\mathbb{R}^{3}\right)\right)}\right\}_{k}\right\|_{\ell^{s}}<\infty .
$$

Obviously, $\widetilde{L}_{T}^{1}\left(\dot{B}_{r, 1}^{\sigma}\right)=L_{T}^{1}\left(\dot{B}_{r, 1}^{\sigma}\right)$.

Next we recall the estimates of the linear transport equation and heat equation in Besov spaces which will be used in the subsequence.

Proposition 2.3. Let $T>0, \sigma \in\left(-3 \min \left(1 / r, 1 / r^{\prime}\right), 1+3 / r\right]$, and $1 \leq r \leq+\infty$. Let $v$ be a vector field so that $\nabla v \in L_{T}^{1}\left(\dot{B}_{r, 1}^{3 / r}\right)$. Assume that $u_{0} \in \dot{B}_{r, 1}^{\sigma}, f \in L_{T}^{1}\left(\dot{B}_{r, 1}^{\sigma}\right)$ and $u$ is the solution of

$$
\left\{\begin{aligned}
\partial_{t} u+v \cdot \nabla u & =f \\
u(0, x) & =u_{0}
\end{aligned}\right.
$$

Then, for $t \in[0, T]$, there holds

$$
\|u\|_{\widetilde{L}_{t}^{\infty} \dot{B}_{r, 1}^{s}} \leq e^{C V(t)}\left(\left\|u_{0}\right\|_{\dot{B}_{r, 1}^{\sigma}}+\int_{0}^{t} e^{-C V(\tau)}\|f(\tau)\|_{\dot{B}_{r, 1}^{\sigma}} d \tau\right),
$$

where $V(t)=\int_{0}^{t}\|\nabla v(\tau)\|_{\dot{B}_{r, 1}^{3 / r}} d \tau$.

Proposition 2.4. Let $T>0, \sigma \in \mathbb{R}$ and $1 \leq r \leq \infty$. Assume that $u_{0} \in \dot{B}_{r, 1}^{\sigma}$ and $f \in \widetilde{L}_{T}^{\rho} \dot{B}_{r, 1}^{\sigma-2+2 / \rho}$. If $u$ is the solution of the heat equation

$$
\left\{\begin{aligned}
\partial_{t} u-\mu \Delta u & =f \\
u(0, x) & =u_{0}
\end{aligned}\right.
$$

with $\mu>0$, then for all $\rho_{1} \in[\rho, \infty]$, it holds that

$$
\mu^{1 / \rho_{1}}\|u\|_{\widetilde{L}_{T}^{\rho_{1}} \dot{B}_{r, 1}^{\sigma+2 / \rho_{1}}} \leq C\left(\left\|u_{0}\right\|_{\dot{B}_{r, 1}^{\sigma}}+\|f\|_{\widetilde{L}_{T}^{\rho} \dot{B}_{r, 1}^{\sigma-2+2 / \rho}}\right) .
$$

We refer to [1] for more details. 


\subsection{Nonlinear estimates in Besov space}

Let us first recall some classical product estimates in Besov spaces from [1].

Lemma 2.5. Let $T>0, \sigma>0$ and $1 \leq r, \rho \leq \infty$. Then it holds that

$$
\|f g\|_{\widetilde{L}_{T}^{\rho} \dot{B}_{r, 1}^{\sigma}} \leq C\left(\|f\|_{L_{T}^{\infty}\left(L^{\infty}\right)}\|g\|_{\widetilde{L}_{T}^{\rho} \dot{B}_{r, 1}^{\sigma}}+\|g\|_{L_{T}^{\infty}\left(L^{\infty}\right)}\|f\|_{\widetilde{L}_{T}^{\rho} \dot{B}_{r, 1}^{\sigma}}\right) .
$$

Lemma 2.6. Let $T>0, \sigma_{1}, \sigma_{2} \leq 3 / r, \sigma_{1}+\sigma_{2}>3 \max (0,2 / r-1)$ and $1 \leq$ $r, \rho, \rho_{1}, \rho_{2} \leq \infty$ with $1 / \rho=1 / \rho_{1}+1 / \rho_{2}$. Then it holds that

$$
\|f g\|_{\widetilde{L}_{T}^{\rho} \dot{B}_{r, 1}^{\sigma_{1}+\sigma_{2}-3 / r}} \leq C\|f\|_{\widetilde{L}_{T}^{\rho_{1}} \dot{B}_{r, 1}^{\sigma_{1}}}\|g\|_{\widetilde{L}_{T}^{\rho_{2}} \dot{B}_{r, 1}^{\sigma_{2}}} .
$$

Lemma 2.7. Let $T>0, \sigma>0$ and $1 \leq r, \rho \leq \infty$. Assume that $F \in W_{l o c}^{[\sigma]+3, \infty}(\mathbb{R})$ with $F(0)=0$. Then for any $f \in L^{\infty} \cap \dot{B}_{r, 1}^{\sigma}$, we have

$$
\|F(f)\|_{\widetilde{L}_{T}^{\rho} \dot{B}_{r, 1}^{\sigma}} \leq C\left(1+\|f\|_{L_{T}^{\infty}\left(L^{\infty}\right)}\right)^{[\sigma]+2}\|f\|_{\widetilde{L}_{T}^{\rho} \dot{B}_{r, 1}^{\sigma}} .
$$

We will use Bony's decomposition

$$
f g=T_{f} g+T_{g} f+R(f, g),
$$

where

$$
T_{f} g=\sum_{j \in \mathbb{Z}} S_{j-1} f \Delta_{j} g, \quad R(f, g)=\sum_{\left|j^{\prime}-j\right| \leq 1} \Delta_{j} f \Delta_{j^{\prime}} g .
$$

We need the following estimates for the paraproduct $T_{f} g$ and $R(f, g)$.

Lemma 2.8. Let $\sigma, \alpha \in \mathbb{R}$ and $1 \leq r, b, \rho, \rho_{1}, \rho_{2} \leq \infty$ with $1 / \rho=1 / \rho_{1}+1 / \rho_{2}$. Then we have

1. if $\alpha \geq 0$, then

$$
\left\|T_{f} g\right\|_{\widetilde{L}_{T}^{\rho} \dot{B}_{b, 1}^{\sigma}} \leq\|f\|_{\widetilde{L}_{T}^{\rho_{1}} \dot{B}_{r, 1}^{3 / r-\alpha}}\|g\|_{\widetilde{L}_{T}^{\rho_{2}} \dot{B}_{b, 1}^{\sigma+\alpha}}
$$

2. if $3 / r-3 / b+\alpha \geq 0$ and $r \geq b$, then

$$
\left\|T_{f} g\right\|_{\widetilde{L}_{T}^{\rho} \dot{B}_{b, 1}^{\sigma}} \leq C\|f\|_{\widetilde{L}_{T}^{\rho_{1}} \dot{B}_{b, 1}^{3 / b-\alpha}}\|g\|_{\widetilde{L}_{T}^{\rho_{2}} \dot{B}_{r, 1}^{\sigma+3 / r-3 / b+\alpha}}
$$

3. if $3 / r+\sigma>0$, then

$$
\|R(f, g)\|_{\widetilde{L}_{T}^{\rho} \dot{B}_{b, 1}^{\sigma}} \leq\|f\|_{\widetilde{L}_{T}^{\rho_{1}} \dot{B}_{r, 1}^{3 / r-\alpha}}\|g\|_{\widetilde{L}_{T}^{\rho_{2}} \dot{B}_{b, 1}^{\sigma+\alpha}} .
$$

Proof. Due to $3 / r-3 / b+\alpha \geq 0$, we infer from Hölder's inequality that

$$
\begin{aligned}
\left\|T_{f} g\right\|_{\widetilde{L}_{T}^{\rho} \dot{B}_{b, 1}^{\sigma}} & \leq \sum_{k \in \mathbb{Z}}\left\|S_{k-1} f\right\|_{L_{T}^{\rho_{2}\left(L^{r-b}\right)}}\left\|\Delta_{k} g\right\|_{L_{T}^{\rho_{1}}\left(L^{r}\right)} 2^{k \sigma} \\
& \leq \sum_{k^{\prime} \leq k-1} 2^{\left(\frac{3}{b}-\alpha\right) k^{\prime}}\left\|\Delta_{k^{\prime}} f\right\|_{L_{T}^{\rho_{1}}\left(L^{b}\right)} 2^{\left(\frac{3}{r}-\frac{3}{b}+\alpha\right) k^{\prime}}\left\|\Delta_{k} g\right\|_{L_{T}^{\rho_{2}}\left(L^{r}\right)} 2^{k \sigma} \\
& \leq C\|f\|_{\widetilde{L}_{T}^{\rho_{1}} \dot{B}_{b, 1}^{3 / b-\alpha}}\|g\|_{\widetilde{L}_{T}^{\rho_{2}} \dot{B}_{r, 1}^{\sigma+3 / r-3 / b+\alpha} .}
\end{aligned}
$$


Here we used the fact that

$$
\left\|\Delta_{k^{\prime}} f\right\|_{L^{r b /(r-b)}} \leq C 2^{3 k^{\prime} / r}\left\|\Delta_{k^{\prime}} f\right\|_{L^{b}},
$$

which can be deduced from Young's inequality. This proves (ii). The proofs of (i) and (iii) are similar.

\section{Ill-posedness of the baratropic Navier-Stokes equations}

\subsection{Reformualtion of the equation}

We introduce the new unknowns

$$
a=\frac{\rho}{\bar{\rho}}-1, \quad h=\Lambda^{-1} \operatorname{div} u, \quad \Omega=\Lambda^{-1} \operatorname{curl} u,
$$

where $\Lambda^{s} f \stackrel{\text { def }}{=} \mathcal{F}^{-1}\left(|\xi|^{s} \hat{f}(\xi)\right)$. The velocity $u$ is decomposed as

$$
u=-\Lambda^{-1} \nabla h+\Lambda^{-1} \operatorname{curl} \Omega .
$$

In terms of new unknown, the system (1.3) can be rewritten as

$$
\left\{\begin{array}{l}
\partial_{t} a+u \cdot \nabla a+\operatorname{div} u(1+a)=0 \\
\partial_{t} h-\bar{\nu} \Delta h=-\Lambda^{-1} \operatorname{div}(u \cdot \nabla u+L(a) \mathcal{A} u+K(a) \nabla a), \\
\partial_{t} \Omega-\bar{\mu} \Delta \Omega=-\Lambda^{-1} \operatorname{curl}(u \cdot \nabla u+L(a) \mathcal{A} u), \\
\left.(a, h, \Omega)\right|_{t=0}=\left(a_{0}, h_{0}, \Omega_{0}\right),
\end{array}\right.
$$

where

$$
\mathcal{A}=\bar{\mu} \Delta+(\bar{\lambda}+\bar{\mu}) \nabla \operatorname{div}, \quad K(a)=\frac{P^{\prime}(\bar{\rho}(1+a))}{1+a} \quad \text { and } \quad L(a)=\frac{a}{1+a}
$$

with $\bar{\mu}=\mu / \bar{\rho}, \bar{\lambda}=\lambda / \bar{\rho}$, and $\bar{\nu}=\bar{\lambda}+2 \bar{\mu}$. Using the Duhanel formula, we obtain

$$
\begin{aligned}
& h(t, x)=e^{\bar{\nu} \Delta t} h_{0}-\int_{0}^{t} e^{\bar{\nu}(t-\tau) \Delta} \Lambda^{-1} \operatorname{div}(u \cdot \nabla u+L(a) \mathcal{A} u+K(a) \nabla a) d \tau, \\
& \Omega(t, x)=e^{\bar{\mu} \Delta t} \Omega_{0}-\int_{0}^{t} e^{\bar{\mu}(t-\tau) \Delta} \Lambda^{-1} \operatorname{curl}(u \cdot \nabla u+L(a) \mathcal{A} u) d \tau .
\end{aligned}
$$

We denote

$$
\begin{aligned}
& U_{0}=-\Lambda^{-2} \nabla \operatorname{div}\left(e^{\bar{\nu} \Delta t} u_{0}\right)+\Lambda^{-2} \operatorname{curl} \operatorname{curl}\left(e^{\bar{\mu} \Delta t} u_{0}\right), \\
& U_{1}=-\Lambda^{-1} \nabla h_{1}+\Lambda^{-1} \operatorname{curl} \Omega_{1}, \\
& U_{2}=-\Lambda^{-1} \nabla h_{2}+\Lambda^{-1} \operatorname{curl} \Omega_{2},
\end{aligned}
$$


where

$$
\begin{aligned}
& h_{1}=-\int_{0}^{t} e^{\bar{\nu}(t-\tau) \Delta} \Lambda^{-1} \operatorname{div}\left(U_{0} \cdot \nabla U_{0}\right)(\tau) d \tau, \\
& \Omega_{1}=-\int_{0}^{t} e^{\bar{\mu}(t-\tau) \Delta} \Lambda^{-1} \operatorname{curl}\left(U_{0} \cdot \nabla U_{0}\right)(\tau) d \tau, \\
& h_{2}=-\int_{0}^{t} e^{\bar{\nu}(t-\tau) \Delta} \Lambda^{-1} \operatorname{div}\left(F_{1}+F_{2}+F_{3}\right)(\tau) d \tau, \\
& \Omega_{2}=-\int_{0}^{t} e^{\bar{\mu}(t-\tau) \Delta} \Lambda^{-1} \operatorname{curl}\left(F_{1}+F_{2}\right)(\tau) d \tau,
\end{aligned}
$$

with $F_{1}, F_{2}, F_{3}$ given by

$$
\begin{aligned}
& F_{1}=U_{0} \cdot \nabla\left(U_{1}+U_{2}\right)+\left(U_{1}+U_{2}\right) \cdot \nabla U_{0}+\left(U_{1}+U_{2}\right) \cdot \nabla\left(U_{1}+U_{2}\right), \\
& F_{2}=L(a) \mathcal{A} u, \quad F_{3}=K(a) \nabla a .
\end{aligned}
$$

Hence, we have

$$
u=-\Lambda^{-1} \nabla h+\Lambda^{-1} \operatorname{curl} \Omega=U_{0}+U_{1}+U_{2} .
$$

\subsection{The choice of initial data}

Choose $\phi$ as a smooth, radial and non-negative function in $\mathbb{R}^{3}$ such that

$$
\phi(\xi)=\left\{\begin{array}{lll}
1 & \text { for } & |\xi| \leq 1 \\
0 & \text { for } & |\xi| \geq 2
\end{array}\right.
$$

Let $N \in \mathbb{N}$, to be determined later. We construct the initial data $\left(\rho_{0}, u_{0}\right)$ as

$$
\begin{aligned}
a_{0} & =\frac{\rho_{0}}{\bar{\rho}}-1=\frac{1}{C(N)}\left(\mathcal{F}^{-1} \phi\right)(x), \\
\widehat{u}_{0}(\xi) & =\frac{2^{N(1-3 / p)}}{C(N)}\left(\phi\left(\xi-2^{N} \tilde{e}\right)+\phi\left(\xi+2^{N} \tilde{e}\right), i \phi\left(\xi-2^{N} \tilde{e}\right)-i \phi\left(\xi+2^{N} \tilde{e}\right), 0\right),
\end{aligned}
$$

where $\tilde{e}=(1,1,0)$ and $C(N)=2^{N 2(3 / q-3 / p+\epsilon)}$ for some $\epsilon>0, q>3, p>6$. Obviously, the initial velocity $u_{0}$ is a real-valued field.

The following lemma can be easily verified.

Lemma 3.1. Let $p>6$. There exist $\epsilon>0$ and $(\widetilde{p}, q)$ satisfying

$$
\begin{aligned}
& 3<q<6, \quad 6<\tilde{p}<p, \quad \frac{3}{\widetilde{p}}+\frac{3}{q}-1>0, \\
& \max \left(\frac{2}{\widetilde{p}}-\frac{1}{q}-\frac{1}{p}, \frac{3}{5 q}-\frac{3}{5 p}\right)<\epsilon<\frac{1}{3}-\frac{1}{q}-\frac{1}{p} .
\end{aligned}
$$

In the sequel, we will fix such a triplet $(\epsilon, \widetilde{p}, q)$. It is easy to verify that

$$
\left\|a_{0}\right\|_{\dot{B}_{q, 1}^{3 / q}} \leq \frac{C}{C(N)}, \quad\left\|u_{0}\right\|_{\dot{B}_{p, 1}^{-1+3 / p}} \leq \frac{C}{C(N)} .
$$


Here and in what follows, we denote by $C$ a constant independent of $N$. Moreover, for any $r \in[1, \infty]$ and $\gamma>3 / p-1$, we have

$$
\left\|u_{0}\right\|_{\dot{B}_{r, 1}^{\gamma}} \leq \frac{C 2^{N(\gamma-3 / p+1)}}{C(N)},
$$

which along with Proposition 2.4 gives

$$
\begin{aligned}
\left\|U_{0}\right\|_{\widetilde{L}_{T}^{\rho} \dot{B}_{r, 1}^{\gamma}} & \leq C T^{1 / \rho_{1}}\left\|U_{0}\right\| \widetilde{L}_{T}^{\rho_{2} \dot{B}_{r, 1}^{\gamma}} \leq C T^{1 / \rho_{1}}\left\|u_{0}\right\|_{\dot{B}_{r, 1}^{\gamma-2 / \rho_{2}}} \\
& \leq C T^{1 / \rho_{1}} \frac{2^{N\left(\gamma-3 / p+1-2 / \rho_{2}\right)}}{C(N)}
\end{aligned}
$$

for any $r, \rho, \rho_{1}, \rho_{2} \in[1, \infty]$ and $\gamma>3 / p-1+2 / \rho_{2}$ with $1 / \rho=1 / \rho_{1}+1 / \rho_{2}$.

\subsection{The lower bound estimate of $\left\|U_{1}\right\|_{\dot{B}_{p, 1}^{3 / p-1}}$}

Since $\dot{B}_{p, 1}^{3 / p-1} \hookrightarrow \dot{B}_{\infty, \infty}^{-1}$, we have

$$
\begin{aligned}
\left\|U_{1}(t)\right\|_{\dot{B}_{p, 1}^{3 / p-1}} & \geq\left\|U_{1}(t)\right\|_{\dot{B}_{\infty}^{-1}, \infty}=\sup _{j \in \mathbb{Z}} 2^{-j}\left\|\Delta_{j} U_{1}(t)\right\|_{\infty} \geq c\left\|\Delta_{-4} U_{1}(t)\right\|_{L^{\infty}} \\
& =c\left\|\int_{\mathbb{R}^{3}} e^{i x \xi} \varphi\left(2^{4} \xi\right) \widehat{U_{1}}(t, \xi) d \xi\right\|_{L_{x}^{\infty}} \geq c\left|\int_{\mathbb{R}^{3}} \varphi\left(2^{4} \xi\right) \widehat{U_{1}}(t, \xi) d \xi\right|
\end{aligned}
$$

for some $c>0$ independent of $N$, where $\varphi$ comes from the Littlewood-Paley decomposition. Set

$$
\begin{aligned}
& \mathfrak{U}_{11}=\iint_{0}^{t} \varphi\left(2^{4} \xi\right) e^{-\bar{\nu}(t-\tau)|\xi|^{2}} \mathcal{F}\left(U_{0} \cdot \nabla U_{0}\right)(\tau, \xi) d \tau d \xi \\
& \mathfrak{U}_{12}=\iint_{0}^{t} \frac{\varphi\left(2^{4} \xi\right)}{|\xi|^{2}}\left(e^{-\bar{\mu}(t-\tau)|\xi|^{2}}-e^{-\bar{\nu}(t-\tau)|\xi|^{2}}\right) \mathcal{F}\left(\operatorname{curl} \operatorname{curl}\left(U_{0} \cdot \nabla U_{0}\right)\right)(\tau, \xi) d \tau d \xi
\end{aligned}
$$

We have

$$
\left|\int \varphi\left(2^{4} \xi\right) \widehat{U_{1}}(t, \xi) d \xi\right| \geq\left|\mathfrak{U}_{11}+\mathfrak{U}_{12}\right| .
$$

Due to div curl $u=0$, we rewrite $U_{0} \cdot \nabla U_{0}$ as

$$
\begin{aligned}
U_{0} \cdot \nabla U_{0}= & \frac{1}{2} \nabla\left|\Lambda^{-2} \nabla \operatorname{div} e^{\bar{\nu} \Delta \tau} u_{0}\right|^{2}+\operatorname{div}\left(\Lambda^{-2} \operatorname{curl} \operatorname{curl} e^{\bar{\mu} \Delta \tau} u_{0} \otimes U_{0}\right) \\
& -\Lambda^{-2} \nabla \operatorname{div} e^{\bar{\nu} \Delta \tau} u_{0} \cdot \nabla \Lambda^{-2} \operatorname{curl} \operatorname{curl} e^{\bar{\mu} \Delta \tau} u_{0}
\end{aligned}
$$

and decompose $\mathfrak{U}_{11}$ into

$$
\mathfrak{U}_{11}=\mathfrak{U}_{11}^{1}+\mathfrak{U}_{11}^{2},
$$


where

$$
\begin{aligned}
\mathfrak{U}_{11}^{1}= & \iint_{0}^{t} \varphi\left(2^{4} \xi\right) e^{-\bar{\nu}(t-\tau)|\xi|^{2}} \mathcal{F}\left(\Lambda^{-2} \nabla \operatorname{div} e^{\bar{\nu} \Delta \tau} u_{0} \cdot \nabla \Lambda^{-2} \operatorname{curl} \operatorname{curl} e^{\bar{\mu} \Delta \tau} u_{0}\right)(\xi) d \tau d \xi, \\
\mathfrak{U}_{11}^{2}= & \iint_{0}^{t} \varphi\left(2^{4} \xi\right) e^{-\bar{\nu}(t-\tau)|\xi|^{2}}\left\{\frac{1}{2} \mathcal{F}\left(\nabla\left|\Lambda^{-2} \nabla \operatorname{div} e^{\bar{\nu} \Delta \tau} u_{0}\right|^{2}\right)(\xi)\right. \\
& \left.+\mathcal{F}\left(\operatorname{div}\left(\Lambda^{-2} \operatorname{curlcurl} e^{\bar{\mu} \Delta \tau} u_{0} \otimes U_{0}\right)\right)(\xi)\right\} d \tau d \xi .
\end{aligned}
$$

It follows from (3.5) and (3.6) that

$$
\left\|U_{1}(t)\right\|_{\dot{B}_{p, 1}^{3 / p-1}} \geq\left|\mathfrak{U}_{11}^{1}\right|-\left|\mathfrak{U}_{11}^{2}\right|-\left|\mathfrak{U}_{12}\right| .
$$

In what follows, we consider the case $t \leq 2^{-2 N}$.

\section{- The estimate of $\mathfrak{U}_{11}^{2}$.}

It follows from Lemma 2.6, Proposition 2.4 and (3.3) that

$$
\begin{aligned}
\left|\mathfrak{U}_{11}^{2}\right| \leq & \| \int_{0}^{t} e^{-\bar{\nu}(t-\tau) \Delta}\left\{\frac{1}{2} \nabla\left|\Lambda^{-2} \nabla \operatorname{div} e^{\bar{\nu} \Delta \tau} u_{0}\right|^{2}\right. \\
& \left.+\operatorname{div}\left(\Lambda^{-2} \operatorname{curl} \operatorname{curl} e^{\bar{\mu} \Delta \tau} u_{0} \otimes U_{0}\right)\right\} d \tau \|_{\dot{B}_{\infty, \infty}^{-1}} \\
\leq & C\left\|\left|\Lambda^{-2} \nabla \operatorname{div} e^{\bar{\nu} \Delta \tau} u_{0}\right|^{2}+\Lambda^{-2} \operatorname{curl} \operatorname{curl} e^{\bar{\mu} \Delta \tau} u_{0} \otimes U_{0}\right\|_{L_{t}^{1} \dot{B}_{\infty, 1}^{0}} \\
\leq & C\left\|\left|\Lambda^{-2} \nabla \operatorname{div} e^{\bar{\nu} \Delta \tau} u_{0}\right|^{2}+\Lambda^{-2} \operatorname{curl} \operatorname{curl} e^{\bar{\mu} \Delta \tau} u_{0} \otimes U_{0}\right\|_{L_{t}^{1} \dot{B}_{p, 1}^{3 / p}} \\
\leq & C\left\|e^{\bar{\nu} \Delta \tau} u_{0}\right\|_{\widetilde{L}_{t}^{2} \dot{B}_{p, 1}^{3 / p}}^{2}+C\left\|e^{\bar{\mu} \Delta \tau} u_{0}\right\|_{\widetilde{L}_{t}^{2} \dot{B}_{p, 1}^{3 / p}}^{2} \leq C\left\|u_{0}\right\|_{\dot{B}_{p, 1}^{3 / p-1}}^{2} \leq \frac{C}{C(N)^{2}} .
\end{aligned}
$$

\section{- The estimate of $\mathfrak{U}_{12}$.}

Using (3.7), we decompose $\mathfrak{U}_{12}$ as

$$
\mathfrak{U}_{12}=\mathfrak{U}_{12}^{1}+\mathfrak{U}_{12}^{2},
$$

where

$$
\begin{aligned}
\mathfrak{U}_{12}^{1}= & \iint_{0}^{t} \varphi\left(2^{4} \xi\right)\left(e^{-\bar{\mu}(t-\tau)|\xi|^{2}}-e^{-\bar{\nu}(t-\tau)|\xi|^{2}}\right)|\xi|^{-2} \\
& \times \mathcal{F}\left(\operatorname{curl} \operatorname{curl}\left(\Lambda^{-2} \nabla \operatorname{div} e^{\bar{\nu} \Delta \tau} u_{0} \cdot \nabla \Lambda^{-2} \operatorname{curl} \operatorname{curl} e^{\bar{\mu} \Delta \tau} u_{0}\right)\right)(\xi) d \tau d \xi \\
\mathfrak{U}_{12}^{2}= & \iint_{0}^{t} \varphi\left(2^{4} \xi\right)\left(e^{-\bar{\mu}(t-\tau)|\xi|^{2}}-e^{-\bar{\nu}(t-\tau)|\xi|^{2}}\right)|\xi|^{-2} \\
& \quad \times \mathcal{F}\left(\operatorname { c u r l } \operatorname { c u r l } \left\{\frac{1}{2} \nabla\left|\Lambda^{-2} \nabla \operatorname{div} e^{\bar{\nu} \Delta \tau} u_{0}\right|^{2}\right.\right. \\
& \left.\left.+\operatorname{div}\left(\Lambda^{-2} \operatorname{curlcurl} e^{\bar{\mu} \Delta \tau} u_{0} \otimes U_{0}\right)\right\}\right)(\xi) d \tau d \xi .
\end{aligned}
$$


By the same argument as the one deriving (3.9), we infer

$$
\left|\mathfrak{U}_{12}^{2}\right| \leq C\left\|u_{0}\right\|_{\dot{B}_{p, 1}^{3 / p-1}}^{2} \leq \frac{C}{C(N)^{2}}
$$

We denote by $a(\xi)$ the symbol of the operator curlcurl. Then $\mathfrak{U}_{12}^{1}$ is written as

$$
\begin{aligned}
\iint_{0}^{t} \varphi\left(2^{4} \xi\right)|\xi|^{-2} & \left(e^{-\bar{\mu}(t-\tau)|\xi|^{2}}-e^{-\bar{\nu}(t-\tau)|\xi|^{2}}\right) \int \frac{e^{-\bar{\nu} \tau|\eta|^{2}}}{|\eta|^{2}} \frac{e^{-\bar{\mu} \tau|\xi-\eta|^{2}}}{|\xi-\eta|^{2}} \\
& \times a(\xi)\left(\mathcal{F}\left(\nabla \operatorname{div} u_{0}\right)(\eta) \cdot \mathcal{F}\left(\nabla \operatorname{curl} \operatorname{curl} u_{0}\right)(\xi-\eta)\right) d \eta d \tau d \xi
\end{aligned}
$$

Due to the choice of $u_{0}$, we find that $|\eta| \gg|\xi| \sim 1$, and this yields

$$
\begin{aligned}
& \int_{0}^{t}\left(e^{-\bar{\mu}(t-\tau)|\xi|^{2}}-e^{-\bar{\nu}(t-\tau)|\xi|^{2}}\right) e^{-\bar{\nu} \tau|\eta|^{2}-\bar{\mu} \tau|\xi-\eta|^{2}} d \tau \\
& \quad=\frac{e^{-\bar{\mu} t|\xi|^{2}}-e^{-\bar{\nu} t|\eta|^{2}-\bar{\mu} t|\xi-\eta|^{2}}}{\bar{\nu}|\eta|^{2}+\bar{\mu}|\xi-\eta|^{2}-\bar{\mu}|\xi|^{2}}-\frac{e^{-\bar{\nu} t|\xi|^{2}}-e^{-\bar{\nu} t|\eta|^{2}-\bar{\mu} t|\xi-\eta|^{2}}}{\bar{\nu}|\eta|^{2}+\bar{\mu}|\xi-\eta|^{2}-\bar{\nu}|\xi|^{2}} \leq C t^{2}|\eta|^{2}
\end{aligned}
$$

Then we obtain

$$
\left|\mathfrak{U}_{12}^{1}\right| \leq \frac{C t^{2}}{C(N)^{2}} 2^{(5-6 / p) N} .
$$

This, along with (3.10), implies that

$$
\left|\mathfrak{U}_{12}\right| \leq \frac{C}{C(N)^{2}}+\frac{C 2^{(5-6 / p) N} t^{2}}{C(N)^{2}} .
$$

- The estimate of $\mathfrak{U}_{11}^{1}$.

The $\ell^{\prime}$-th component $\mathfrak{U}_{11}^{1 \ell^{\prime}}$ of $\mathfrak{U}_{11}^{1}$ is given by

$$
\begin{aligned}
\mathfrak{U}_{11}^{1, \ell^{\prime}}= & \iint_{0}^{t} \varphi\left(2^{4} \xi\right) e^{-\bar{\nu}(t-\tau)|\xi|^{2}} i \int \frac{\eta_{\ell} \eta_{m}}{|\eta|^{2}|\xi-\eta|^{2}} e^{-\bar{\nu} \tau|\eta|^{2}} \widehat{u}_{0 m}(\eta)(\xi-\eta)_{\ell} e^{-\bar{\mu} \tau|\xi-\eta|^{2}} \\
& \times(\xi-\eta)_{m^{\prime}}\left((\xi-\eta)_{m^{\prime}} \widehat{u}_{0 \ell^{\prime}}(\xi-\eta)-(\xi-\eta)_{\ell^{\prime}} \widehat{u}_{0 m^{\prime}}(\xi-\eta)\right) d \eta d \tau d \xi \\
\triangleq & \iint_{0}^{t} \varphi\left(2^{4} \xi\right) e^{-\bar{\nu}(t-\tau)|\xi|^{2}} i A(t, \xi) d \tau d \xi .
\end{aligned}
$$

In what follows, we consider the case of $\ell^{\prime}=1$. From the construction of $u_{0}$, we find that

$$
A(\tau, \xi)=\frac{1}{C(N)^{2}} 2^{2 N(1-3 / p)}\left(A_{1}+A_{2}\right),
$$


where

$$
\begin{aligned}
A_{1}=\int & {\left[\eta_{1}\left(\xi_{2}-\eta_{2}\right)^{2}+\eta_{1}\left(\xi_{3}-\eta_{3}\right)^{2}-\eta_{2}\left(\xi_{1}-\eta_{1}\right)\left(\xi_{2}-\eta_{2}\right)+i \eta_{1}\left(\xi_{1}-\eta_{1}\right)\left(\xi_{2}-\eta_{2}\right)\right.} \\
& \left.+i \eta_{2}\left(\xi_{2}-\eta_{2}\right)^{2}+i \eta_{2}\left(\xi_{3}-\eta_{3}\right)^{2}\right] \frac{\eta_{\ell}\left(\xi_{\ell}-\eta_{\ell}\right)}{|\eta|^{2}|\xi-\eta|^{2}} e^{-\bar{\nu} \tau|\eta|^{2}-\bar{\mu} \tau|\xi-\eta|^{2}} \\
& \times \phi\left(\eta-2^{N} \tilde{e}\right), \phi\left(\xi-\eta+2^{N} \tilde{e}\right) d \eta \\
A_{2}=\int & {\left[\eta_{1}\left(\xi_{2}-\eta_{2}\right)^{2}+\eta_{1}\left(\xi_{3}-\eta_{3}\right)^{2}-\eta_{2}\left(\xi_{1}-\eta_{1}\right)\left(\xi_{2}-\eta_{2}\right)-i \eta_{1}\left(\xi_{1}-\eta_{1}\right)\left(\xi_{2}-\eta_{2}\right)\right.} \\
& \left.-i \eta_{2}\left(\xi_{2}-\eta_{2}\right)^{2}-i \eta_{2}\left(\xi_{3}-\eta_{3}\right)^{2}\right] \frac{\eta_{\ell}\left(\xi_{\ell}-\eta_{\ell}\right)}{|\eta|^{2}|\xi-\eta|^{2}} e^{-\bar{\nu} \tau|\eta|^{2}-\bar{\mu} \tau|\xi-\eta|^{2}} \\
& \times \phi\left(\eta+2^{N} \tilde{e}\right) \phi\left(\xi-\eta-2^{N} \tilde{e}\right) d \eta .
\end{aligned}
$$

Making a change of variable, we obtain

$$
\begin{aligned}
A_{1}=\int & {\left[\left(\eta_{1}+2^{N}\right)\left(\left(\xi_{2}-\eta_{2}-2^{N}\right)^{2}+\left(\xi_{3}-\eta_{3}\right)^{2}\right)\right.} \\
& -\left(\eta_{2}+2^{N}\right)\left(\xi_{1}-\eta_{1}-2^{N}\right)\left(\xi_{2}-\eta_{2}-2^{N}\right) \\
& +i\left(\eta_{1}+2^{N}\right)\left(\xi_{1}-\eta_{1}-2^{N}\right)\left(\xi_{2}-\eta_{2}-2^{N}\right) \\
& \left.+i\left(\eta_{2}+2^{N}\right)\left(\left(\xi_{2}-\eta_{2}-2^{N}\right)^{2}+\left(\xi_{3}-\eta_{3}\right)^{2}\right)\right] \\
& \times \frac{\left(\eta+2^{N} \tilde{e}\right) \ell\left(\xi-\eta-2^{N} \tilde{e}\right) \ell}{\left|\eta+2^{N} \tilde{e}\right|^{2}\left|\xi-\eta-2^{N} \tilde{e}\right|^{2}} e^{-\bar{\nu} \tau\left|\eta+2^{N} \tilde{e}\right|^{2}-\bar{\mu} \tau\left|\xi-\eta-2^{N} \tilde{e}\right|^{2}} \phi(\eta) \phi(\xi-\eta) d \eta,
\end{aligned}
$$

and

$$
\begin{aligned}
A_{2}=\int & {\left[\left(\eta_{1}-2^{N}\right)\left(\left(\xi_{2}-\eta_{2}+2^{N}\right)^{2}+\left(\xi_{3}-\eta_{3}\right)^{2}\right)\right.} \\
& -\left(\eta_{2}-2^{N}\right)\left(\xi_{1}-\eta_{1}+2^{N}\right)\left(\xi_{2}-\eta_{2}+2^{N}\right) \\
& -i\left(\eta_{1}-2^{N}\right)\left(\xi_{1}-\eta_{1}+2^{N}\right)\left(\xi_{2}-\eta_{2}+2^{N}\right) \\
& \left.-i\left(\eta_{2}-2^{N}\right)\left(\left(\xi_{2}-\eta_{2}+2^{N}\right)^{2}+\left(\xi_{3}-\eta_{3}\right)^{2}\right)\right] \\
& \times \frac{\left(\eta-2^{N} \tilde{e}\right) \ell\left(\xi-\eta+2^{N} \tilde{e}\right) \ell}{\left|\eta-2^{N} \tilde{e}\right|^{2}\left|\xi-\eta+2^{N} \tilde{e}\right|^{2}} e^{-\bar{\nu} \tau\left|\eta-2^{N} \tilde{e}\right|^{2}-\bar{\mu} \tau\left|\xi-\eta+2^{N} \tilde{e}\right|^{2} \phi(\eta) \phi(\xi-\eta) d \eta .}
\end{aligned}
$$

Due to the choice of $\phi$, we find

$$
\begin{aligned}
& A_{1}=\int\left(-i 2^{2+5 N}+O\left(2^{4 N}\right)\right) \frac{e^{-\bar{\nu} \tau\left|\eta+2^{N} \tilde{e}\right|^{2}-\bar{\mu} \tau\left|\xi-\eta-2^{N} \tilde{e}\right|^{2}}}{\left|\eta+2^{N} \tilde{e}\right|^{2}\left|\xi-\eta-2^{N} \tilde{e}\right|^{2}} \phi(\eta) \phi(\xi-\eta) d \eta \\
& A_{2}=\int\left(-i 2^{2+5 N}+O\left(2^{4 N}\right)\right) \frac{e^{-\bar{\nu} \tau\left|\eta-2^{N} \tilde{e}\right|^{2}-\bar{\mu} \tau\left|\xi-\eta+2^{N} \tilde{e}\right|^{2}}}{\left|\eta-2^{N} \tilde{e}\right|^{2}\left|\xi-\eta+2^{N} \tilde{e}\right|^{2}} \phi(\eta) \phi(\xi-\eta) d \eta .
\end{aligned}
$$

This yields that

$$
\mathfrak{U}_{11}^{1,1}=\mathfrak{U}_{11}^{11,1}+\mathfrak{U}_{11}^{12,1},
$$


where

$$
\begin{aligned}
\mathfrak{U}_{11}^{11,1}= & \frac{1}{C(N)^{2}} 2^{2 N(1-3 / p)} \iint_{0}^{t} \varphi\left(2^{4} \xi\right) e^{-\bar{\nu}(t-\tau)|\xi|^{2}} \int 2^{2+5 N} \phi(\eta) \phi(\xi-\eta) \\
& \times\left\{\frac{e^{-\bar{\nu} \tau\left|\eta+2^{N} \tilde{e}\right|^{2}-\bar{\mu} \tau\left|\xi-\eta-2^{N} \tilde{e}\right|^{2}}}{\left|\eta+2^{N} \tilde{e}\right|^{2}\left|\xi-\eta-2^{N} \tilde{e}\right|^{2}}+\frac{e^{-\bar{\nu} \tau\left|\eta-2^{N} \tilde{e}\right|^{2}-\bar{\mu} \tau\left|\xi-\eta+2^{N} \tilde{e}\right|^{2}}}{\left|\eta-2^{N} \tilde{e}\right|^{2}\left|\xi-\eta+2^{N} \tilde{e}\right|^{2}}\right\} d \eta d \tau d \xi, \\
\mathfrak{U}_{11}^{12,1}= & \frac{1}{C(N)^{2}} 2^{2 N(1-3 / p)} \iint_{0}^{t} \varphi\left(2^{4} \xi\right) e^{-\bar{\nu}(t-\tau)|\xi|^{2}} i \int O\left(2^{4 N}\right) \phi(\eta) \phi(\xi-\eta) \\
& \times\left\{\frac{e^{-\bar{\nu} \tau\left|\eta+2^{N} \tilde{e}\right|^{2}-\bar{\mu} \tau\left|\xi-\eta-2^{N} \tilde{e}\right|^{2}}}{\left|\eta+2^{N} \tilde{e}\right|^{2}\left|\xi-\eta-2^{N} \tilde{e}\right|^{2}}+\frac{e^{-\bar{\nu} \tau\left|\eta-2^{N} \tilde{e}\right|^{2}-\bar{\mu} \tau\left|\xi-\eta+2^{N} \tilde{e}\right|^{2}}}{\left|\eta-2^{N} \tilde{e}\right|^{2}\left|\xi-\eta+2^{N} \tilde{e}\right|^{2}}\right\} d \eta d \tau d \xi .
\end{aligned}
$$

After integrating with respect to $\tau$, we get

$$
\begin{aligned}
\mathfrak{U}_{11}^{11,1}= & \frac{4}{C(N)^{2}} 2^{2 N(1-3 / p)} \int \varphi\left(2^{4} \xi\right) \int 2^{5 N} \phi(\eta) \phi(\xi-\eta) \\
& \times\left\{\frac{e^{-\bar{\nu} t|\xi|^{2}}-e^{-\bar{\nu} t\left|\eta+2^{N} \tilde{e}\right|^{2}-\bar{\mu} t\left|\xi-\eta-2^{N} \tilde{e}\right|^{2}}}{\left|\eta+2^{N} \tilde{e}\right|^{2}\left|\xi-\eta-2^{N} \tilde{e}\right|^{2}\left(\bar{\nu}\left|\eta+2^{N} \tilde{e}\right|^{2}+\bar{\mu}\left|\xi-\eta-2^{N} \tilde{e}\right|^{2}-\bar{\nu}|\xi|^{2}\right)}\right. \\
& \left.+\frac{e^{-\bar{\nu} t|\xi|^{2}}-e^{-\bar{\nu} t\left|\eta-2^{N} \tilde{e}\right|^{2}-\bar{\mu} t\left|\xi-\eta+2^{N} \tilde{e}\right|^{2}}}{\left|\eta-2^{N} \tilde{e}\right|^{2}\left|\xi-\eta+2^{N} \tilde{e}\right|^{2}\left(\bar{\nu}\left|\eta-2^{N} \tilde{e}\right|^{2}+\bar{\mu}\left|\xi-\eta+2^{N} \tilde{e}\right|^{2}-\bar{\nu}|\xi|^{2}\right)}\right\} d \eta d \xi .
\end{aligned}
$$

Using Taylor's formula, we infer

$$
\begin{aligned}
& \frac{e^{-\bar{\nu} t|\xi|^{2}}-e^{-\bar{\nu} t\left|\eta+2^{N} \tilde{e}\right|^{2}-\bar{\mu} t\left|\xi-\eta-2^{N} \tilde{e}\right|^{2}}}{\bar{\nu}\left|\eta+2^{N} \tilde{e}\right|^{2}+\bar{\mu}\left|\xi-\eta-2^{N} \tilde{e}\right|^{2}-\bar{\nu}|\xi|^{2}}=t+O\left(t^{2} 2^{2 N}\right), \\
& \frac{e^{-\bar{\nu} t|\xi|^{2}}-e^{-\bar{\nu} t\left|\eta-2^{N} \tilde{e}\right|^{2}-\bar{\mu} t\left|\xi-\eta+2^{N} \tilde{e}\right|^{2}}}{\bar{\nu}\left|\eta-2^{N} \tilde{e}\right|^{2}+\bar{\mu}\left|\xi-\eta+2^{N} \tilde{e}\right|^{2}-\bar{\nu}|\xi|^{2}}=t+O\left(t^{2} 2^{2 N}\right),
\end{aligned}
$$

from which it follows that

$$
\begin{aligned}
\mathfrak{U}_{11}^{11,1} & =\frac{4}{C(N)^{2}} 2^{2 N(1-3 / p)} \iint \varphi\left(2^{4} \xi\right) 2^{5 N} \phi(\eta) \phi(\xi-\eta) \\
& \times\left\{\frac{t}{\left|\eta+2^{N} \tilde{e}\right|^{2}\left|\xi-\eta-2^{N} \tilde{e}\right|^{2}}+\frac{t}{\left|\eta-2^{N} \tilde{e}\right|^{2}\left|\xi-\eta+2^{N} \tilde{e}\right|^{2}}+O\left(t^{2} 2^{-2 N}\right)\right\} d \eta d \xi
\end{aligned}
$$

Hence,

$$
\mathfrak{U}_{11}^{11,1} \geq \frac{c t 2^{3 N-6 N / p}}{C(N)^{2}}-\frac{C t^{2} 2^{5 N-6 N / p}}{C(N)^{2}} .
$$

Similarly, we can deduce

$$
\begin{aligned}
\left|\mathfrak{U}_{11}^{12,1}\right| \leq & \frac{C}{C(N)^{2}} \iint 2^{2 N(1-3 / p)}\left\{\frac{e^{-\bar{\nu} t|\xi|^{2}}-e^{-\bar{\nu} t\left|\eta+2^{N} \tilde{e}\right|^{2}-\bar{\mu} t\left|\xi-\eta-2^{N} \tilde{e}\right|^{2}}}{\bar{\nu}\left|\eta+2^{N} \tilde{e}\right|^{2}+\bar{\mu}\left|\xi-\eta-2^{N} \tilde{e}\right|^{2}-\bar{\nu}|\xi|^{2}}\right. \\
& \left.+\frac{e^{-\bar{\nu} t|\xi|^{2}}-e^{-\bar{\nu} t\left|\eta-2^{N} \tilde{e}\right|^{2}-\bar{\mu} t\left|\xi-\eta+2^{N} \tilde{e}\right|^{2}}}{\bar{\nu}\left|\eta-2^{N} \tilde{e}\right|^{2}+\bar{\mu}\left|\xi-\eta+2^{N} \tilde{e}\right|^{2}-\bar{\nu}|\xi|^{2}}\right\} \phi(\eta) \phi(\xi-\eta) d \eta d \xi \\
\leq & \frac{C}{C(N)^{2}} 2^{-6 N / p} \leq \frac{C}{C(N)^{2}} .
\end{aligned}
$$


Hence, we conclude that

$$
\left|\mathfrak{U}_{11}^{1}\right| \geq\left|\mathfrak{U}_{11}^{11}\right| \geq \frac{c t 2^{3 N-6 N / p}}{C(N)^{2}}-\frac{C t^{2} 2^{5 N-6 N / p}}{C(N)^{2}}-\frac{C}{C(N)^{2}} .
$$

Summing up (3.8), (3.9), (3.11) and (3.14), we obtain

$$
\left\|U_{1}(t)\right\|_{\dot{B}_{p, 1}^{3 / p-1}} \geq \frac{c t 2^{3 N-6 N / p}}{C(N)^{2}}-\frac{C t^{2} 2^{5 N-6 N / p}}{C(N)^{2}}-\frac{C N^{2}}{C(N)^{2}} .
$$

Choosing $t=2^{-2(1+\epsilon) N}$ and recalling $C(N)^{2}=2^{N(3 / q-3 / p+\varepsilon)}$, we get

$$
\left\|U_{1}(t)\right\|_{\dot{B}_{p, 1}^{3 / p-1}} \geq c 2^{(1-3 / q-3 / p-3 \epsilon) N}
$$

for some $c>0$ independent of $N$.

\subsection{The estimate of $\left\|U_{1}\right\|_{L_{T}^{1} \dot{B}_{q, 1}^{3 / q+1} \cap \widetilde{L}_{T}^{2} \dot{B}_{q, 1}^{3 / q}}$}

Let $(p, q, \widetilde{p})$ be given as in Lemma 3.1. It follows from Hölder's inequality and Proposition 2.4 that

$$
\begin{aligned}
\left\|U_{1}\right\|_{L_{T}^{1} \dot{B}_{q, 1}^{3 / q+1}} & +\left\|U_{1}\right\|_{\widetilde{L}_{T}^{2} \dot{B}_{q, 1}^{3 / q}} \\
& \leq C\left(\left\|h_{1}\right\|_{L_{T}^{1} \dot{B}_{q, 1}^{3 / q+1}}+\left\|h_{1}\right\|_{\widetilde{L}_{T}^{2} \dot{B}_{q, 1}^{3 / q}}+\left\|\Omega_{1}\right\|_{L_{T}^{1} \dot{B}_{q, 1}^{3 / q+1}}+\left\|\Omega_{1}\right\|_{\widetilde{L}_{T}^{2} \dot{B}_{q, 1}^{3 / q}}\right) \\
& \leq C T^{1 / 2}\left(\left\|h_{1}\right\|_{\widetilde{L}_{T}^{2} \dot{B}_{q, 1}^{3 / q+1}}+\left\|\Omega_{1}\right\|_{\widetilde{L}_{T}^{2} \dot{B}_{q, 1}^{3 / q+1}}+\left\|h_{1}\right\|_{\widetilde{L}_{T}^{\infty} \dot{B}_{q, 1}^{3 / q}}+\left\|\Omega_{1}\right\|_{\widetilde{L}_{T}^{\infty} \dot{B}_{q, 1}^{3 / q}}\right) \\
& \leq C T^{1 / 2}\left\|U_{0} \cdot \nabla U_{0}\right\|_{L_{T}^{1} \dot{B}_{q, 1}^{3 / q}} .
\end{aligned}
$$

We infer from Lemma $2.5, \dot{B}_{p, 1}^{3 / p} \hookrightarrow L^{\infty}$ and (3.4) that

$$
\begin{aligned}
\left\|U_{0} \cdot \nabla U_{0}\right\|_{L_{T}^{1} \dot{B}_{q, 1}^{3 / q}} & \leq C\left(\left\|U_{0}\right\|_{L_{T}^{\infty} L^{\infty}}\left\|\nabla U_{0}\right\|_{L_{T}^{1} \dot{B}_{q, 1}^{3 / q}}+\left\|U_{0}\right\|_{L_{T}^{1} \dot{B}_{q, 1}^{3 / q}}\left\|\nabla U_{0}\right\|_{L_{T}^{\infty} L^{\infty}}\right) \\
& \leq C T^{1 / 2}\left(\left\|U_{0}\right\|_{L_{T}^{\infty} \dot{B}_{p, 1}^{3 / p}}\left\|U_{0}\right\|_{\widetilde{L}_{T}^{2} \dot{B}_{q, 1}^{3 / q+1}}+\left\|U_{0}\right\|_{\widetilde{L}_{T}^{2} \dot{B}_{q, 1}^{3 / q}}\left\|U_{0}\right\|_{L_{T}^{\infty} \dot{B}_{p, 1}^{3 / p+1}}\right) \\
& \leq C T^{1 / 2} \frac{2^{N(3 / q-3 / p+2)}}{C(N)^{2}} .
\end{aligned}
$$

From this, and (3.16), it follows that

$$
\left\|U_{1}\right\|_{L_{T}^{1} \dot{B}_{q, 1}^{3 / q+1}}+\left\|U_{1}\right\|_{\widetilde{L}_{T}^{2} \dot{B}_{q, 1}^{3 / q}} \leq C T \frac{2^{N(3 / q-3 / p+2)}}{C(N)^{2}} .
$$

Similarly, we have

$$
\begin{aligned}
& \left\|U_{1}\right\|_{L_{T}^{1} \dot{B}_{p, 1}^{3 / p+1}}+\left\|U_{1}\right\|_{\widetilde{L}_{T}^{2} \dot{B}_{p, 1}^{3 / p}} \leq C T \frac{2^{2 N}}{C(N)^{2}}, \\
& \left\|U_{1}\right\|_{L_{T}^{1} \dot{B}_{\widetilde{p}, 1}^{3 / \widetilde{p}+1}}+\left\|U_{1}\right\|_{\widetilde{L}_{T}^{2} \dot{B}_{\widetilde{p}, 1}^{3 / \widetilde{p}}} \leq C T \frac{2^{N(3 / \widetilde{p}-3 / p+2)}}{C(N)^{2}} .
\end{aligned}
$$




\subsection{The estimates of $F_{i}(i=1,2,3)$}

Recalling that

$$
F_{3}=K(a) \nabla a=(K(a)-1) \nabla a+\nabla a .
$$

Then it follows from Lemma 2.6 and Lemma 2.7 that

$$
\begin{aligned}
\left\|F_{3}\right\|_{L_{T}^{1} \dot{B}_{q, 1}^{3 / q-1}} & \leq C T\|K(a)-1\|_{L_{T}^{\infty} \dot{B}_{q, 1}^{3 / q}}\|\nabla a\|_{L_{T}^{\infty} \dot{B}_{q, 1}^{3 / q-1}}+\|a\|_{L_{T}^{1} \dot{B}_{q, 1}^{3 / q}} \\
& \leq C T\left(1+\|a\|_{L_{T}^{\infty}\left(L^{\infty}\right)}\right)^{2}\|a\|_{L_{T}^{\infty} \dot{B}_{q, 1}^{3 / q}}^{2}+C T\|a\|_{L_{T}^{\infty} \dot{B}_{q, 1}^{3 / q}}
\end{aligned}
$$

We write $F_{2}$ as

$$
F_{2}=L(a) \mathcal{A} u=L(a) \mathcal{A}\left(U_{0}+U_{1}\right)+L(a) \mathcal{A} U_{2} .
$$

By making use of Lemma 2.6 again, (3.4) and (3.17), we obtain

$$
\begin{aligned}
\left\|L(a) \mathcal{A}\left(U_{0}+U_{1}\right)\right\|_{L_{T}^{1} \dot{B}_{q, 1}^{3 / q-1}} & \leq C\|L(a)\|_{L_{T}^{\infty} \dot{B}_{q, 1}^{3 / q}}\left\|\mathcal{A}\left(U_{0}+U_{1}\right)\right\|_{L_{T}^{1} \dot{B}_{q, 1}^{3 / q-1}} \\
& \leq C\left(\|a\|_{L_{T}^{\infty}\left(L^{\infty}\right)}+1\right)^{2}\|a\|_{L_{T}^{\infty} \dot{B}_{q, 1}^{3 / q}}\left\|U_{0}+U_{1}\right\|_{L_{T}^{1} \dot{B}_{q, 1}^{3 / q+1}} \\
& \leq C\left(\|a\|_{L_{T}^{\infty}\left(L^{\infty}\right)}+1\right)^{2}\|a\|_{L_{T}^{\infty} \dot{B}_{q, 1}^{3 / q}} \frac{2^{N(3 / q-3 / p+2)}}{C(N)},
\end{aligned}
$$

and

$$
\begin{aligned}
\left\|L(a) \mathcal{A} U_{2}\right\|_{L_{T}^{1} \dot{B}_{q, 1}^{3 / q-1}} & \leq C\|L(a)\|_{L_{T}^{\infty} \dot{B}_{q, 1}^{3 / q}}\left\|\mathcal{A} U_{2}\right\|_{L_{T}^{1} \dot{B}_{q, 1}^{3 / q-1}} \\
& \leq C\left(\|a\|_{L_{T}^{\infty}\left(L^{\infty}\right)}+1\right)^{2}\|a\|_{L_{T}^{\infty} \dot{B}_{q, 1}^{3 / q}}\left\|U_{2}\right\|_{L_{T}^{1} \dot{B}_{q, 1}^{3 / q+1}}
\end{aligned}
$$

This above two estimates give that

$$
\begin{aligned}
\left\|F_{2}\right\|_{L_{T}^{1} \dot{B}_{q, 1}^{3 / q-1}} \leq & C\left(\|a\|_{L_{T}^{\infty}\left(L^{\infty}\right)}+1\right)^{2}\|a\|_{L_{T}^{\infty} \dot{B}_{q, 1}^{3 / q}} \\
& \times\left(T \frac{2^{N(3 / q-3 / p+2)}}{C(N)}+\left\|U_{2}\right\|_{L_{T}^{1} \dot{B}_{q, 1}^{3 / q+1}}\right) .
\end{aligned}
$$

Now let us turn to the estimate of $F_{1}$, which is given by

$$
F_{1}=U_{0} \cdot \nabla\left(U_{1}+U_{2}\right)+\left(U_{1}+U_{2}\right) \cdot \nabla U_{0}+\left(U_{1}+U_{2}\right) \cdot \nabla\left(U_{1}+U_{2}\right) .
$$

Due to $3 / p-3 / q+1>0$ and $3 / \widetilde{p}+3 / q-1>0$, we apply $(2.1)$, Lemma 2.8 , (3.4) and (3.17) to get

$$
\begin{aligned}
& \left\|U_{0} \cdot \nabla\left(U_{1}+U_{2}\right)\right\|_{L_{T}^{1} \dot{B}_{q, 1}^{3 / q-1}} \\
& \quad \leq C\left\|U_{0}\right\|_{\widetilde{L}_{T}^{2} \dot{B}_{p, 1}^{3 / p}}\left\|\nabla\left(U_{1}+U_{2}\right)\right\|_{\widetilde{L}_{T}^{2} \dot{B}_{q, 1}^{3 / q-1}}+C\left\|U_{0}\right\|_{\widetilde{L}_{T}^{2} \dot{B}_{\widetilde{p}, 1}^{3 / \widetilde{p}}}\left\|\nabla\left(U_{1}+U_{2}\right)\right\|_{\widetilde{L}_{T}^{2} \dot{B}_{q, 1}^{3 / q-1}} \\
& (3.22) \leq C T^{1 / 2}\left(\frac{2^{N}}{C(N)}+\frac{2^{N(3 / \widetilde{p}-3 / p+1)}}{C(N)}\right)\left(T \frac{2^{N(3 / q-3 / p+2)}}{C(N)^{2}}+\left\|U_{2}\right\|_{\widetilde{L}_{T}^{2} \dot{B}_{q, 1}^{3 / q}}\right),
\end{aligned}
$$


and

$$
\begin{aligned}
\left\|U_{1} \cdot \nabla U_{0}\right\|_{L_{T}^{1} \dot{B}_{q, 1}^{3 / q-1}} \leq C( & \left\|\nabla U_{0}\right\|_{\widetilde{L}_{T}^{2} \dot{B}_{p, 1}^{3 / p-1}}\left\|U_{1}\right\|_{\widetilde{L}_{T}^{2} \dot{B}_{q, 1}^{3 / q}} \\
& +\left\|\nabla U_{0}\right\|_{\widetilde{L}_{T}^{2} \dot{B}_{q, 1}^{3 / q-1}}\left\|U_{1}\right\|_{\widetilde{L}_{T}^{2} \dot{B}_{p, 1}^{3 / p}} \\
& \left.+\left\|\nabla U_{0}\right\|_{\widetilde{L}_{T}^{2} \dot{B}_{\widetilde{p}, 1}^{3 / \widetilde{p}-1}}\left\|U_{1}\right\|_{\widetilde{L}_{T}^{2} \dot{B}_{q, 1}^{3 / q}}\right) \\
\leq C T^{3 / 2} & \left(\frac{2^{N(3 / q-3 / p+3)}}{C(N)^{3}}+\frac{2^{N(3 / \widetilde{p}+3 / q-6 / p+3)}}{C(N)^{3}}\right),
\end{aligned}
$$

and

$$
\begin{gathered}
\left\|U_{2} \cdot \nabla U_{0}\right\|_{L_{T}^{1} \dot{B}_{q, 1}^{3 / q-1}} \leq C\left(\left\|\nabla U_{0}\right\|_{\widetilde{L}_{T}^{2} \dot{B}_{p, 1}^{3 / p-1}}\left\|U_{2}\right\|_{\widetilde{L}_{T}^{2} \dot{B}_{q, 1}^{3 / q}}\right. \\
+\left\|\nabla U_{0}\right\|_{L_{T}^{1} \dot{B}_{p, 1}^{3 / p}}\left\|U_{2}\right\|_{\widetilde{L}_{T}^{\infty} \dot{B}_{q, 1}^{3 / q-1}} \\
\left.+\left\|\nabla U_{0}\right\|_{\widetilde{L}_{T}^{2} \dot{B}_{\widetilde{p}, 1}^{3 / \widetilde{p}-1}}\left\|U_{2}\right\|_{\widetilde{L}_{T}^{2} \dot{B}_{q, 1}^{3 / q}}\right) \\
(3.24) \leq C T^{1 / 2}\left(\frac{2^{N}}{C(N)}+\frac{2^{N(3 / \widetilde{p}-3 / p+1)}}{C(N)}\right)\left\|U_{2}\right\|_{\widetilde{L}_{T}^{2} \dot{B}_{q, 1}^{3 / q}}+C T \frac{2^{2 N}}{C(N)}\left\|U_{2}\right\|_{\widetilde{L}_{T}^{\infty} \dot{B}_{q, 1}^{3 / q-1}} .
\end{gathered}
$$

By Lemma 2.8 and (3.17)-(3.19), we have

$$
\begin{aligned}
&\left\|U_{1} \cdot \nabla\left(U_{1}+U_{2}\right)\right\|_{L_{T}^{1} \dot{B}_{q, 1}^{3 / q-1}}+\left\|U_{2} \cdot \nabla U_{1}\right\|_{L_{T}^{1} \dot{B}_{q, 1}^{3 / q-1}} \\
& \leq C\left\|U_{1}\right\|_{\widetilde{L}_{T}^{2} \dot{B}_{p, 1}^{3 / p}}\left\|\nabla\left(U_{1}+U_{2}\right)\right\|_{\widetilde{L}_{T}^{2} \dot{B}_{q, 1}^{3 / q-1}} \\
&+C\left\|U_{1}\right\|_{\widetilde{L}_{T}^{2} \dot{B}_{\widetilde{p}, 1}^{3 / \widetilde{p}}}\left\|\nabla\left(U_{1}+U_{2}\right)\right\|_{\widetilde{L}_{T}^{2} \dot{B}_{q, 1}^{3 / q-1}} \\
&+C\left\|\nabla U_{1}\right\|_{L_{T}^{1} \dot{B}_{p, 1}^{3 / p}}\left\|U_{2}\right\|_{\widetilde{L}_{T}^{\infty} \dot{B}_{q, 1}^{3 / q-1}}+\left\|\nabla U_{1}\right\|_{L_{T}^{1} \dot{B}_{\widetilde{p}, 1}^{3 / \widetilde{p}}}\left\|U_{2}\right\|_{\widetilde{L}_{T}^{\infty} \dot{B}_{q, 1}^{3 / q-1}} \\
& \leq C T^{2}\left(\frac{2^{N(3 / q-3 / p+4)}}{C(N)^{4}}+\frac{2^{N(3 / \widetilde{p}+3 / q-6 / p+4)}}{C(N)^{4}}\right) \\
&+C T\left(\frac{2^{2 N}}{C(N)^{2}}+\frac{2^{N(3 / \widetilde{p}-3 / p+2)}}{C(N)^{2}}\right)\left\|U_{2}\right\|_{\widetilde{L}_{T}^{2} \dot{B}_{q, 1}^{3 / q}} \\
&+C T\left(\frac{2^{2 N}}{C(N)^{2}}+\frac{2^{N(3 / \widetilde{p}-3 / p+2)}}{C(N)^{2}}\right)\left\|U_{2}\right\|_{\widetilde{L}_{T}^{\infty} \dot{B}_{q, 1}^{3 / q-1}} .
\end{aligned}
$$

We infer from Lemma 2.6 that

$$
\left\|U_{2} \cdot \nabla U_{2}\right\|_{L_{T}^{1} \dot{B}_{q, 1}^{3 / q-1}} \leq C\left\|U_{2}\right\|_{\widetilde{L}_{T}^{2} \dot{B}_{q, 1}^{3 / q}}\left\|\nabla U_{2}\right\|_{\widetilde{L}_{T}^{2} \dot{B}_{q, 1}^{3 / q-1}} \leq C\left\|U_{2}\right\|_{\widetilde{L}_{T}^{2} \dot{B}_{q, 1}^{3 / q}}^{2} \cdot
$$


Summing up (3.22)-(3.26), we obtain

$$
\begin{aligned}
\left\|F_{1}\right\|_{L_{T}^{1} \dot{B}_{q, 1}^{3 / q-1}} \leq & \frac{C T^{3 / 2}}{C(N)^{3}}\left(2^{N(3 / q-3 / p+3)}+2^{N(3 / \widetilde{p}+3 / q-6 / p+3)}\right) \\
& +\frac{C T^{2}}{C(N)^{4}}\left(2^{N(3 / q-3 / p+4)}+2^{N(3 / \widetilde{p}+3 / q-6 / p+4)}\right)+C\left\|U_{2}\right\|_{\widetilde{L}_{T}^{2} \dot{B}_{q, 1}^{3 / q}}^{2} \\
& +\left\{\frac{C T^{1 / 2}}{C(N)}\left(2^{N}+2^{N(3 / \widetilde{p}-3 / p+1)}\right)\right. \\
& \left.+C T\left(\frac{2^{2 N}}{C(N)}+\frac{2^{N(3 / \widetilde{p}-3 / p+2)}}{C(N)^{2}}\right)\right\}\left\|U_{2}\right\|_{\widetilde{L}_{T}^{2} \dot{B}_{q, 1}^{3 / q}} \\
& +\frac{C T}{C(N)}\left(\frac{2^{2 N}}{C(N)}+\frac{2^{N(3 / \widetilde{p}-3 / p+2)}}{C(N)}+2^{2 N}\right)\left\|U_{2}\right\|_{\widetilde{L}_{T}^{\infty} \dot{B}_{q, 1}^{3 / q-1} .} .
\end{aligned}
$$

\subsection{Proof of Theorem 1.1}

We denote

$$
X_{T}=\|a\|_{\widetilde{L}_{T}^{\infty} \dot{B}_{q, 1}^{3 / q}}, \quad Y_{T}=\left\|U_{2}\right\|_{\widetilde{L}_{T}^{\infty} \dot{B}_{q, 1}^{3 / q-1}}+\left\|U_{2}\right\|_{L_{T}^{1} \dot{B}_{q, 1}^{3 / q+1}}
$$

For $T \leq T_{0}=2^{-2(1+\epsilon) N}$, it follows from Proposition 2.4, (3.20), (3.21) and (3.27) that

$$
\begin{aligned}
Y_{T} \leq & C \sum_{i=1}^{3}\left\|F_{i}\right\|_{L_{T}^{1} \dot{B}_{q, 1}^{3 / q-1}} \\
\leq & \frac{C T^{3 / 2} 2^{N(3 / \widetilde{p}+3 / q-6 / p+3)}}{C(N)^{3}}+\frac{C T^{1 / 2} 2^{N(3 / \widetilde{p}-3 / p+1)}}{C(N)} Y_{T}+C Y_{T}^{2} \\
& +\frac{C T 2^{N(3 / q-3 / p+2)}}{C(N)}\left(1+X_{T}\right)^{3} X_{T}+C\left(1+X_{T}\right)^{2} X_{T} Y_{T}
\end{aligned}
$$

On the other hand, we infer from Proposition 2.3 that

$$
X_{T} \leq C \exp \left(\|\nabla u\|_{L_{T}^{1} \dot{B}_{p, 1}^{3 / p}}\right)\left(\left\|a_{0}\right\|_{\dot{B}_{q, 1}^{3 / q}}+\|\operatorname{div} u+a \operatorname{div} u\|_{L_{T}^{1} \dot{B}_{q, 1}^{3 / q}}\right) .
$$

Thanks to Lemma 2.6, (3.4) and (3.17), we have

$$
\begin{aligned}
\|a \operatorname{div} u\|_{L_{T}^{1} \dot{B}_{q, 1}^{3 / q}} & \leq C\left\|\operatorname{div}\left(U_{0}+U_{1}\right)\right\|_{L_{T}^{1} \dot{B}_{q, 1}^{3 / q}} X_{T}+C Y_{T} X_{T} \\
& \leq \frac{C T 2^{N(3 / q-3 / p+2)}}{C(N)} X_{T}+C Y_{T} X_{T} .
\end{aligned}
$$

This gives by (3.4) and (3.17) that

(3.29) $X_{T} \leq C \exp \left(\frac{C T 2^{2 N}}{C(N)}+C Y_{T}\right)\left(\frac{1+T 2^{N\left(\frac{3}{q}-\frac{3}{p}+2\right)}}{C(N)}\left(1+X_{T}\right)+Y_{T}+X_{T} Y_{T}\right)$. 
Due to $\max (2 / \widetilde{p}-1 / q-1 / p, 3 /(5 q)-3 /(5 p))<\epsilon$, we can take $N$ big enough such that

$$
\frac{C T^{1 / 2} 2^{N(3 / \widetilde{p}-3 / p+1)}}{C(N)} \ll 1, \quad \frac{C T 2^{N(3 / q-3 / p+2)}}{C(N)} \ll 1
$$

for any $T \leq T_{0}$. By using of the continuation argument, we deduce from (3.28) and (3.29) that for any $T \leq T_{0}$,

$$
X_{T} \leq \frac{C\left(1+T 2^{N(3 / q-3 / p+2)}\right)}{C(N)}, \quad Y_{T} \leq \frac{C T 2^{N(3 / q-3 / p+2)}}{C(N)} .
$$

Summing up (3.4), (3.15) and (3.30), we conclude that

$$
\begin{aligned}
\left\|u\left(T_{0}\right)\right\|_{\dot{B}_{p, 1}^{3 / p-1}} & \geq\left\|U_{1}\left(T_{0}\right)\right\|_{\dot{B}_{p, 1}^{3 / p-1}}-\left\|U_{0}\left(T_{0}\right)\right\|_{\dot{B}_{p, 1}^{3 / p-1}}-\left\|U_{2}\left(T_{0}\right)\right\|_{\dot{B}_{p, 1}^{3 / p-1}} \\
& \geq c 2^{N(1-3 / q-3 / p-3 \epsilon)}-C 2^{-\frac{N}{2}(3 / q-3 / p+\epsilon)}-C 2^{N(3 /(2 q)-3 /(2 p)-5 / 2 \epsilon)} \\
& \geq \frac{c}{2} 2^{N(1-3 / q-3 / p-3 \epsilon)}
\end{aligned}
$$

if $N$ is taken sufficiently large, as $1-3 / q-3 / p-3 \epsilon>0$. This completes the proof of Theorem 1.1.

\section{Ill-posedness of the heat-conductive flows}

\subsection{Reformulation of the equation}

We denote

$$
a=\frac{\rho}{\bar{\rho}}-1, \quad u=U_{0}+\widetilde{U},
$$

where $U_{0}$ is defined as in (3.4). Then the system (1.2) can be rewritten as

$$
\left\{\begin{array}{l}
\partial_{t} a+u \cdot \nabla a+\operatorname{div} u(1+a)=0 \\
\partial_{t} \widetilde{U}-\mathcal{A} \widetilde{U}=-u \cdot \nabla u-L(a) \mathcal{A} u-R \nabla \theta-R \nabla a \theta+R \nabla a L(a) \theta \\
\partial_{t} \theta+u \cdot \nabla \theta-\tilde{\kappa} \Delta \theta+\tilde{\kappa} L(a) \Delta \theta+\tilde{R} \theta \operatorname{div} u \\
\quad=\frac{\tilde{\mu}}{2(a+1)}\left|\nabla u+(\nabla u)^{\top}\right|^{2}+\frac{\tilde{\lambda}}{a+1}|\operatorname{div} u|^{2} \\
\left.(a, \widetilde{U}, \theta)\right|_{t=0}=\left(a_{0}, 0, \theta_{0}\right)
\end{array}\right.
$$

where $\tilde{\kappa}=\frac{\kappa}{c_{V} \bar{\rho}}, \tilde{R}=\frac{R}{c_{V}}, \tilde{\mu}=\frac{\mu}{c_{V} \bar{\rho}}, \tilde{\lambda}=\frac{\lambda}{c_{V} \bar{\rho}}$, and

$$
\mathcal{A}=\bar{\mu} \Delta+(\bar{\lambda}+\bar{\mu}) \nabla \operatorname{div}, \quad L(a)=\frac{a}{1+a} .
$$

We decompose $\theta$ into

$$
\theta(x, t)=\Theta_{0}+\theta_{1}+\theta_{2},
$$


where

$$
\begin{aligned}
\Theta_{0}= & e^{\tilde{\kappa} \Delta t} \theta \\
\theta_{1}= & \int_{0}^{t} e^{\tilde{\kappa} \Delta(t-\tau)}\left(\frac{\tilde{\mu}}{2}\left|\nabla U_{0}+\left(\nabla U_{0}\right)^{\top}\right|^{2}+\tilde{\lambda}\left|\operatorname{div} U_{0}\right|^{2}\right) d \tau, \\
\theta_{2}= & \int_{0}^{t} e^{\tilde{\kappa} \Delta(t-\tau)}\left(\frac{\tilde{\mu}}{2}\left|\nabla \widetilde{U}+(\nabla \widetilde{U})^{\top}\right|^{2}+\tilde{\mu}\left(\nabla U_{0}+\left(\nabla U_{0}\right)^{\top}\right):\left(\nabla \widetilde{U}+(\nabla \widetilde{U})^{\top}\right)\right. \\
& +\tilde{\lambda}\left(|\operatorname{div} \widetilde{U}|^{2}+2 \operatorname{div} U_{0} \operatorname{div} \widetilde{U}\right)-u \cdot \nabla \theta-\tilde{\kappa} L(a) \Delta \theta \\
& \left.-\tilde{R} \theta \operatorname{div} u-\frac{\tilde{\mu}}{2} L(a)\left|\nabla u+(\nabla u)^{\top}\right|^{2}-\tilde{\lambda} L(a)|\operatorname{div} u|^{2}\right) d \tau .
\end{aligned}
$$

\subsection{The choice of initial data}

Let $\phi$ be as in (3.2) and $N \in \mathbb{N}$ be determined later. The initial velocity is chosen as

$$
\widehat{u}_{0}(\xi)=\frac{1}{C(N)} 2^{N(1-3 / p)}\left(\phi\left(\xi-2^{N} e_{1}\right)+\phi\left(\xi+2^{N} e_{1}\right), 0,0\right),
$$

and the initial density and temperature is chosen as

$$
a_{0}=\frac{\rho_{0}}{\bar{\rho}}-1=\frac{1}{2^{N} C(N)} \mathcal{F}^{-1}(\phi)(x), \quad \theta_{0}=\frac{1}{C(N)} \mathcal{F}^{-1}(\phi)(x) .
$$

Here $e_{1}=(1,0,0)$ and $C(N)=2^{\frac{N}{2}(3 / q-3 / p+\epsilon)}$.

Lemma 4.1. Let $p>3$. There exist $\epsilon>0$ and $(\widetilde{p}, q)$ satisfying

$$
\begin{aligned}
& 2<q<3, \quad 3<\widetilde{p}<p, \quad \frac{3}{\widetilde{p}}+\frac{3}{q}-2>0, \\
& \max \left\{\frac{2}{\widetilde{p}}-\frac{1}{q}-\frac{1}{p}, \frac{3}{5}\left(\frac{1}{q}-\frac{1}{p}\right)\right\}<\epsilon<\frac{2}{3}-\frac{1}{q}-\frac{1}{p} .
\end{aligned}
$$

In the sequel, we will fix such a triplet $(\epsilon, \widetilde{p}, q)$. It is easy to check that

$$
\left\|a_{0}\right\|_{\dot{B}_{q, 1}^{3 / q}}+\left\|\theta_{0}\right\|_{\dot{B}_{q, 1}^{-2+3 / q}} \leq \frac{C}{C(N)}, \quad\left\|u_{0}\right\|_{\dot{B}_{p, 1}^{-1+3 / p}} \leq \frac{C}{C(N)} .
$$

Furthermore, it holds that

$$
\left\|U_{0}\right\|_{\widetilde{L}_{T}^{\rho} \dot{B}_{r, 1}^{\sigma}} \leq C T^{1 / \rho_{1}} \frac{2^{N\left(\sigma-3 / p+1-2 / \rho_{2}\right)}}{C(N)}
$$

for any $r, \rho, \rho_{1}, \rho_{2} \in[1, \infty]$ and $\sigma>3 / p-1+2 / \rho_{2}$ with $1 / \rho=1 / \rho_{1}+1 / \rho_{2}$.

\subsection{The lower bound estimate of $\left\|\theta_{1}\right\|_{\dot{B}_{p, 1}^{3 / p-2}}$}

By the same argument as the one used to derive (3.5) and (3.6), we have

$$
\left\|\theta_{1}(t)\right\|_{\dot{B}_{p, 1}^{3 / p-2}} \geq c\left|\int_{\mathbb{R}^{3}} \varphi\left(2^{4} \xi\right) \widehat{\theta}_{1}(t, \xi) d \xi\right| \geq c\left|\theta_{11}+\theta_{12}\right|
$$


where $\theta_{11}$ and $\theta_{12}$ are given by

$$
\begin{aligned}
& \theta_{11}=\tilde{\lambda} \iint_{0}^{t} \varphi\left(2^{4} \xi\right) e^{-\tilde{\kappa}(t-\tau)|\xi|^{2}} \mathcal{F}\left(\left|\operatorname{div} U_{0}\right|^{2}\right)(\tau, \xi) d \tau d \xi \\
& \theta_{12}=\frac{\tilde{\mu}}{2} \iint_{0}^{t} \varphi\left(2^{4} \xi\right) e^{-\tilde{\kappa}(t-\tau)|\xi|^{2}} \mathcal{F}\left(\left|\nabla U_{0}+\left(\nabla U_{0}\right)^{\top}\right|^{2}\right)(\tau, \xi) d \tau d \xi .
\end{aligned}
$$

\section{- The estimate of $\theta_{11}$.}

Recalling the definition of $U_{0}$, we get

$$
\begin{aligned}
\theta_{11}= & -\tilde{\lambda} \iiint_{0}^{t} \varphi\left(2^{4} \xi\right) e^{-\tilde{\kappa} t|\xi|^{2}} e^{\tilde{\kappa} \tau|\xi|^{2}-\bar{\nu} \tau|\xi-\eta|^{2}-\bar{\nu} \tau|\eta|^{2}}(\xi-\eta)_{j} \\
& \times{\widehat{u_{0}}}^{j}(\xi-\eta) \eta_{\ell}{\widehat{u_{0}}}^{\ell}(\eta) d \eta d \tau d \xi \\
= & \frac{\tilde{\lambda}}{C(N)^{2}} 2^{2(1-3 / p) N} \iint \varphi\left(2^{4} \xi\right) \frac{e^{-\bar{\kappa}|\xi|^{2} t}-e^{\left(-\bar{\nu}|\xi-\eta|^{2}-\bar{\nu}|\eta|^{2}\right) t}}{\bar{\kappa}|\xi|^{2}-\bar{\nu}|\xi-\eta|^{2}-\bar{\nu}|\eta|^{2}}\left(\xi_{1}-\eta_{1}\right) \eta_{1} \\
& \times\left(\phi\left(\xi-\eta+2^{N} e_{1}\right) \phi\left(\eta-2^{N} e_{1}\right)+\phi\left(\xi-\eta-2^{N} e_{1}\right) \phi\left(\eta+2^{N} e_{1}\right)\right) d \eta d \xi .
\end{aligned}
$$

Making a change of variable, we find that

$$
\begin{aligned}
\theta_{11}= & \frac{\tilde{\lambda}}{C(N)^{2}} 2^{2(1-3 / p)} N \iint \varphi\left(2^{4} \xi\right) \frac{e^{-\bar{\kappa}|\xi|^{2} t}-e^{\left(-\bar{\nu}\left|\xi-\eta-2^{N} e_{1}\right|^{2}-\bar{\nu}\left|\eta+2^{N} e_{1}\right|^{2}\right) t}}{\bar{\kappa}|\xi|^{2}-\bar{\nu}\left|\xi-\eta-2^{N} e_{1}\right|^{2}-\bar{\nu}\left|\eta+2^{N} e_{1}\right|^{2}} \\
& \times\left(\xi_{1}-\eta_{1}-2^{N}\right)\left(\eta_{1}+2^{N}\right) \phi(\xi-\eta) \phi(\eta) d \eta d \xi \\
& +\frac{\tilde{\lambda}}{C(N)^{2}} 2^{2(1-3 / p) N} \iint \varphi\left(2^{4} \xi\right) \frac{e^{-\bar{\kappa}|\xi|^{2} t}-e^{\left(-\bar{\nu}\left|\xi-\eta+2^{N} e_{1}\right|^{2}-\bar{\nu}\left|\eta-2^{N} e_{1}\right|^{2}\right) t}}{\bar{\kappa}|\xi|^{2}-\bar{\nu}\left|\xi-\eta+2^{N} e_{1}\right|^{2}-\bar{\nu}\left|\eta-2^{N} e_{1}\right|^{2}} \\
& \times\left(\xi_{1}-\eta_{1}+2^{N}\right)\left(\eta_{1}-2^{N}\right) \phi(\xi-\eta) \phi(\eta) d \eta d \xi .
\end{aligned}
$$

Using Taylor's formula, we deduce

$$
\begin{aligned}
\theta_{11}(t)=\frac{2 \tilde{\lambda}}{C(N)^{2}} 2^{2(1-3 / p) N} \iint & \varphi\left(2^{4} \xi\right)\left(-t+O\left(2^{2 N} t^{2}\right)\right) \\
& \times\left(-2^{2 N}+O\left(2^{N}\right)\right) \phi(\xi-\eta) \phi(\eta) d \eta d \xi
\end{aligned}
$$

\section{- The estimate of $\theta_{12}$.}

Noticing that

$$
\mathcal{F}\left(\nabla U_{0}+\left(\nabla U_{0}\right)^{\top}\right)(\eta)=-\left(\widehat{H}_{\ell m}^{1}(\eta)+\widehat{H}_{\ell m}^{2}(\eta)+\widehat{H}_{\ell m}^{3}(\eta)\right),
$$

where $\widehat{H}_{\ell m}^{i}(i=1,2,3)$ is given by

$$
\begin{aligned}
\widehat{H}_{\ell m}^{1}(\eta) & =\frac{2 i}{|\eta|^{2}} e^{-\bar{\nu}|\eta|^{2} \tau} \eta_{\ell} \eta_{m} \eta_{j} \widehat{u}_{0}^{j}(\eta), \\
\widehat{H}_{\ell m}^{2}(\eta) & =-\frac{2 i}{|\eta|^{2}} e^{-\bar{\mu}|\eta|^{2} \tau} \eta_{\ell} \eta_{m} \eta_{j} \widehat{u}_{0}^{j}(\eta), \\
\widehat{H}_{\ell m}^{3}(\eta) & =\frac{i}{|\eta|^{2}} e^{-\bar{\mu}|\eta|^{2} \tau} \eta_{j}^{2}\left(\eta_{\ell} \widehat{u}_{0}^{m}(\eta)+\eta_{m} \widehat{u}_{0}^{\ell}(\eta)\right),
\end{aligned}
$$


we write $\theta_{12}$ as

$\theta_{12}=\frac{\tilde{\mu}}{2} \sum_{J, J^{\prime}=1}^{3} \iiint_{0}^{t} \varphi\left(2^{4} \xi\right) e^{-\tilde{\kappa}(t-\tau)|\xi|^{2}} \widehat{H}_{\ell m}^{J}(\xi-\eta) \widehat{H}_{\ell m}^{J^{\prime}}(\eta) d \tau d \eta d \xi \triangleq \sum_{J, J^{\prime}=1}^{3} \mathcal{H}^{J J^{\prime}}$.

First of all, we consider $\mathcal{H}^{11}$.

$$
\begin{gathered}
\mathcal{H}^{11}(t)=-2 \tilde{\mu} \iiint_{0}^{t} \varphi\left(2^{4} \xi\right) \frac{e^{-\tilde{\kappa}(t-\tau)|\xi|^{2}}}{|\xi-\eta|^{2}|\eta|^{2}} e^{-\bar{\nu} \tau|\xi-\eta|^{2}-\bar{\nu} \tau|\eta|^{2}}(\xi-\eta)_{m}(\xi-\eta)_{\ell}(\xi-\eta)_{j} \\
\times \eta_{m} \eta_{\ell} \eta_{j^{\prime}} \widehat{u}_{0}^{j}(\xi-\eta) \widehat{u}_{0}^{j^{\prime}}(\eta) d \tau d \eta d \xi
\end{gathered}
$$

Making a change of variable and computing the above integral with respect to the time, we deduce

$$
\begin{aligned}
\mathcal{H}^{11}(t)= & \frac{2 \tilde{\mu}}{C(N)^{2}} 2^{2(1-3 / p) N} \iint \varphi\left(2^{4} \xi\right) \frac{e^{-\tilde{\kappa}|\xi|^{2} t}-e^{\left(-\bar{\nu}\left|\xi-\eta-2^{N} e_{1}\right|^{2}-\bar{\nu}\left|\eta+2^{N} e_{1}\right|^{2}\right) t}}{\tilde{\kappa}|\xi|^{2}-\bar{\nu}\left|\xi-\eta-2^{N} e_{1}\right|^{2}-\bar{\nu}\left|\eta+2^{N} e_{1}\right|^{2}} \\
& \times \frac{\left(\xi-\eta-2^{N} e_{1}\right)_{\ell}}{\left|\xi-\eta-2^{N} e_{1}\right|^{2}} \frac{\left(\eta+2^{N} e_{1}\right)_{\ell}}{\left|\eta+2^{N} e_{1}\right|^{2}}\left(\xi-\eta-2^{N} e_{1}\right)_{m}\left(\eta+2^{N} e_{1}\right)_{m} \\
& \times\left(\xi-\eta-2^{N} e_{1}\right)_{1}\left(\eta+2^{N} e_{1}\right)_{1} \phi(\xi-\eta) \phi(\eta) d \eta d \xi+\text { similar term. }
\end{aligned}
$$

Using Taylor's formula, we infer

$$
\begin{aligned}
\mathcal{H}^{11}(t)=\frac{4 \tilde{\mu}}{C(N)^{2}} 2^{2(1-3 / p) N} \iint & \varphi\left(2^{4} \xi\right)\left(-t+O\left(2^{2 N} t^{2}\right)\right) \\
& \times\left(-2^{2 N}+O\left(2^{N}\right)\right) \phi(\xi-\eta) \phi(\eta) d \eta d \xi .
\end{aligned}
$$

By a direct calculation, we find

$$
\begin{aligned}
\mathcal{H}^{33}(t)+\mathcal{H}^{32}(t)= & -2 \tilde{\mu} \iiint_{0}^{t} \varphi\left(2^{4} \xi\right) \frac{e^{-\tilde{\kappa}(t-\tau)|\xi|^{2}}}{|\xi-\eta|^{2}|\eta|^{2}} e^{-\bar{\mu} \tau|\xi-\eta|^{2}-\bar{\mu} \tau|\eta|^{2}}(\xi-\eta)_{j}^{2} \\
& \times\left\{\left(\eta_{2}^{2}+\eta_{3}^{2}\right)\left(2(\xi-\eta)_{1} \eta_{1}+(\xi-\eta)_{2} \eta_{2}+(\xi-\eta)_{3} \eta_{3}\right)\right. \\
& \left.-\eta_{1}^{2}\left((\xi-\eta)_{2} \eta_{2}+(\xi-\eta)_{3} \eta_{3}\right)\right\} \hat{u}_{0}^{1}(\xi-\eta) \hat{u}_{0}^{1}(\eta) d \tau d \eta d \xi
\end{aligned}
$$

Integrating on time, and making a change of variable, we deduce

$$
\begin{aligned}
\mathcal{H}^{33}(t) & +\mathcal{H}^{32}(t) \\
= & \frac{2}{C(N)^{2}} 2^{2(1-3 / p) N} \iint \varphi\left(2^{4} \xi\right) \frac{e^{-\tilde{\kappa}|\xi|^{2} t}-e^{\left(-\bar{\mu}\left|\xi-\eta-2^{N} e_{1}\right|^{2}-\bar{\mu}\left|\eta+2^{N} e_{1}\right|^{2}\right) t}}{\tilde{\kappa}|\xi|^{2}-\bar{\mu}\left|\xi-\eta-2^{N} e_{1}\right|^{2}-\bar{\mu}\left|\eta+2^{N} e_{1}\right|^{2}} \\
& \times \frac{\left(\xi-\eta-2^{N} e_{1}\right)_{j}^{2}}{\left|\xi-\eta-2^{N} e_{1}\right|^{2}\left|\eta+2^{N} e_{1}\right|^{2}} O\left(2^{2 N}\right) \phi(\xi-\eta) \phi(\eta) d \eta d \xi+\text { similar term. }
\end{aligned}
$$

From this, it follows that

$$
\left|\mathcal{H}^{33}(t)+\mathcal{H}^{32}(t)\right| \leq \frac{C}{C(N)^{2}} 2^{-6 N / p} \leq \frac{C}{C(N)^{2}}
$$


Furthermore, we also have

$$
\begin{aligned}
& \mathcal{H}^{22}(t)+\mathcal{H}^{23}(t) \\
& =-2 \widetilde{\mu} \iiint_{0}^{t} \varphi\left(2^{4} \xi\right) \frac{e^{-\tilde{\kappa}(t-\tau)|\xi|^{2}}}{|\xi-\eta|^{2}|\eta|^{2}} e^{-\bar{\mu} \tau|\xi-\eta|^{2}-\bar{\mu} \tau|\eta|^{2}}(\xi-\eta)_{\ell}(\xi-\eta)_{1} \eta_{\ell} \\
& \quad \times\left\{(\xi-\eta)_{2} \eta_{2} \eta_{1}+(\xi-\eta)_{3} \eta_{3} \eta_{1}-(\xi-\eta)_{1}\left(\eta_{2}^{2}+\eta_{3}^{2}\right)\right\} \widehat{u}_{0}^{1}(\xi-\eta) \widehat{u}_{0}^{1}(\eta) d \tau d \eta d \xi
\end{aligned}
$$

and a similar representation for $\mathcal{H}^{12}(t)+\mathcal{H}^{13}(t)$, hence,

$$
\left|\mathcal{H}^{22}(t)+\mathcal{H}^{23}(t)\right|+\left|\mathcal{H}^{12}(t)+\mathcal{H}^{13}(t)\right| \leq \frac{C}{C(N)^{2}} 2^{-6 N / p} \leq \frac{C}{C(N)^{2}} .
$$

For $\mathcal{H}^{21}(t)+\mathcal{H}^{31}(t)$, we have

$$
\begin{aligned}
& \mathcal{H}^{21}(t)+\mathcal{H}^{31}(t) \\
& =2 \widetilde{\mu} \iiint_{0}^{t} \varphi\left(2^{4} \xi\right) \frac{e^{-\tilde{\kappa}(t-\tau)|\xi|^{2}}}{|\xi-\eta|^{2}|\eta|^{2}} e^{-\bar{\mu} \tau|\xi-\eta|^{2}-\bar{\nu} \tau|\eta|^{2}}(\xi-\eta)_{\ell} \eta_{\ell} \eta_{1}\left\{\left((\xi-\eta)_{2} \eta_{2}\right.\right. \\
& \left.\left.\quad+(\xi-\eta)_{3} \eta_{3}\right)(\xi-\eta)_{1}-\left((\xi-\eta)_{2}^{2}+(\xi-\eta)_{3}^{2}\right) \eta_{1}\right\} \widehat{u}_{0}^{1}(\xi-\eta) \widehat{u}_{0}^{1}(\eta) d \tau d \eta d \xi
\end{aligned}
$$

hence,

$$
\left|\mathcal{H}^{21}(t)+\mathcal{H}^{31}(t)\right| \leq \frac{C}{C(N)^{2}}
$$

Summing up (4.5)-(4.10), we conclude that

$$
\begin{aligned}
\left\|\theta_{1}(t)\right\|_{\dot{B}_{p, 1}^{3 / p-2}} & \geq\left|\theta_{11}+\mathcal{H}^{11}(t)\right|-\left|\sum_{1 \leq J, J^{\prime} \leq 3, J J^{\prime} \neq 11} \mathcal{H}^{J J^{\prime}}(t)\right| \\
& \geq \frac{c 2^{2(2-3 / p) N} t}{C(N)^{2}}-\frac{C\left(1+2^{6(1-1 / p) N} t^{2}+2^{(3-6 / p) N} t\right)}{C(N)^{2}} .
\end{aligned}
$$

As $2-3 / q-3 / p>3 \epsilon$, we can take $t \sim 2^{-2(1+\epsilon) N}$ to obtain

$$
\left\|\theta_{1}(t)\right\|_{\dot{B}_{p, 1}^{3 / p-2}} \geq c 2^{N(2-3 / q-3 / p-3 \epsilon)}
$$

for some $c>0$ independent of $N$.

\subsection{The estimate of $\left\|\theta_{1}\right\|_{L_{T}^{1} \dot{B}_{q, 1}^{3 / q} \cap \widetilde{L}_{T}^{2} \dot{B}_{q, 1}^{3 / q-1}}$}

Since $q<3$, we get by Lemma 2.5 that

$$
\begin{aligned}
\left\|\left(\nabla U_{0}\right)^{2}\right\|_{L_{T}^{1} \dot{B}_{q, 1}^{3 / q-1}} & \leq C\left(\left\|\nabla U_{0}\right\|_{L_{T}^{\infty} L^{\infty}}\left\|\nabla U_{0}\right\|_{L_{T}^{1} \dot{B}_{q, 1}^{3 / q-1}}+\left\|\nabla U_{0}\right\|_{L_{T}^{1} \dot{B}_{q, 1}^{3 / q-1}}\left\|\nabla U_{0}\right\|_{L_{T}^{\infty} L^{\infty}}\right) \\
& \leq C T^{1 / 2}\left\|U_{0}\right\|_{L_{T}^{\infty} \dot{B}_{p, 1}^{3 / p+1}}\left\|U_{0}\right\|_{\widetilde{L}_{T}^{2} \dot{B}_{q, 1}^{3 / q}} .
\end{aligned}
$$


By Hölder's inequality and Proposition 2.4, we get

$$
\begin{aligned}
\left\|\theta_{1}\right\|_{L_{T}^{1} \dot{B}_{q, 1}^{3 / q}} & +\left\|\theta_{1}\right\|_{\widetilde{L}_{T}^{2} \dot{B}_{q, 1}^{3 / q-1}} \leq C\left(T^{1 / 2}\left\|\theta_{1}\right\|_{\widetilde{L}_{T}^{2} \dot{B}_{q, 1}^{3 / q}}+\left\|\theta_{1}\right\|_{\widetilde{L}_{T}^{2} \dot{B}_{q, 1}^{3 / q-1}}\right) \\
& \leq C T^{1 / 2}\left\|\left(\nabla U_{0}\right)^{2}\right\|_{L_{T}^{1} \dot{B}_{q, 1}^{3 / q-1}} \leq C T\left\|U_{0}\right\|_{L_{T}^{\infty} \dot{B}_{p, 1}^{3 / p+1}}\left\|U_{0}\right\|_{\widetilde{L}_{T}^{2} \dot{B}_{q, 1}^{3 / q}}
\end{aligned}
$$

This along with (4.4) gives

$$
\left\|\theta_{1}\right\|_{L_{T}^{1} \dot{B}_{q, 1}^{3 / q}}+\left\|\theta_{1}\right\|_{L_{T}^{2} \dot{B}_{q, 1}^{3 / q-1}} \leq C T \frac{2^{N(3 / q-3 / p+2)}}{C(N)^{2}} .
$$

\subsection{Proof of Theorem $\mathbf{1 . 2}$}

We denote

$$
\begin{aligned}
& X_{T}=\|a\|_{\widetilde{L}_{T}^{\infty} \dot{B}_{q, 1}^{3 / q}}, \quad Y_{T}=\|\widetilde{U}\|_{\widetilde{L}_{T}^{\infty} \dot{B}_{q, 1}^{3 / q-1}}+\|\widetilde{U}\|_{L_{T}^{1} \dot{B}_{q, 1}^{3 / q+1}}, \\
& Z_{T}=\left\|\theta_{2}\right\|_{\widetilde{L}_{T}^{\infty} \dot{B}_{q, 1}^{3 / q-2}}+\left\|\theta_{2}\right\|_{L_{T}^{1} \dot{B}_{q, 1}^{3 / q}} \text {. }
\end{aligned}
$$

Step 1. The estimate of $X_{T}$.

It follows from Proposition 2.3, Lemma 2.5 and (4.4) that

$$
\begin{aligned}
X_{T} & \leq C \exp \left(\|\nabla u\|_{L_{T}^{1} \dot{B}_{p, 1}^{3 / p}}\right)\left(\left\|a_{0}\right\|_{\dot{B}_{q, 1}^{3 / q}}+\left\|\operatorname{div}\left(U_{0}+\widetilde{U}\right)+\operatorname{div}\left(U_{0}+\widetilde{U}\right) a\right\|_{L_{T}^{1} \dot{B}_{q, 1}^{3 / q}}\right) \\
(4.13) & \leq C \exp \left(\frac{T 2^{2 N}}{C(N)}+Y_{T}\right)\left(\frac{1}{C(N)}+\frac{T 2^{N\left(\frac{3}{q}-\frac{3}{p}+2\right)}}{C(N)}\left(1+X_{T}\right)+Y_{T}+Y_{T} X_{T}\right) .
\end{aligned}
$$

Similarly, we have

$$
\begin{aligned}
\|a\|_{\widetilde{L}_{T}^{\infty} \dot{B}_{q, 1}^{3 / q-1}} \leq & C \exp \left(\frac{T 2^{2 N}}{C(N)}+Y_{T}\right)\left(\frac{1}{2^{N} C(N)}\right. \\
& \left.+\frac{T 2^{N(3 / q-3 / p+1)}}{C(N)}\left(1+X_{T}\right)+T Y_{T}+T^{1 / 2} Y_{T} X_{T}\right)
\end{aligned}
$$

Step 2. The estimate of $Y_{T}$.

We infer from Proposition 2.4 that

$$
Y_{T} \leq C\|u \cdot \nabla u+L(a) \mathcal{A} u+R \nabla \theta+R \nabla a \theta-R \nabla a L(a) \theta\|_{L_{T}^{1} \dot{B}_{q, 1}^{3 / q-1}}
$$

By Lemma 2.6 and (4.4), we get

$$
\begin{aligned}
\|L(a) \mathcal{A} u\|_{L_{T}^{1} \dot{B}_{q, 1}^{3 / q-1}} & \leq C\|a\|_{L_{T}^{\infty} \dot{B}_{q, 1}^{3 / q}\left\|\nabla^{2}\left(U_{0}+\widetilde{U}\right)\right\|_{L_{T}^{1} \dot{B}_{q, 1}^{3 / q-1}}} \\
& \leq C X_{T}\left(\frac{T 2^{N(3 / q-3 / p+2)}}{C(N)}+Y_{T}\right) .
\end{aligned}
$$


Using Lemma 2.7, (4.3) and (4.12) yields that

$$
\begin{aligned}
&\|\nabla a \theta\|_{L_{T}^{1} \dot{B}_{q, 1}^{3 / q-1}}+\|\nabla a L(a) \theta\|_{L_{T}^{1} \dot{B}_{q, 1}^{3 / q-1}} \\
& \leq C\|\nabla a\|_{L_{T}^{\infty} \dot{B}_{q, 1}^{3 / q-1}}\|\theta\|_{L_{T}^{1} \dot{B}_{q, 1}^{3 / q}}+C\|\nabla a\|_{L_{T}^{\infty} \dot{B}_{q, 1}^{3 / q-1}}\|L(a)\|_{L_{T}^{\infty} \dot{B}_{q, 1}^{3 / q}}\|\theta\|_{L_{T}^{1} \dot{B}_{q, 1}^{3 / q}} \\
&(4.17) \quad \leq C X_{T}\left(1+X_{T}\right)^{3}\left(\frac{1}{C(N)}+\frac{T 2^{N(3 / q-3 / p+2)}}{C(N)^{2}}+Z_{T}\right) .
\end{aligned}
$$

On the other hand, by (2.1), Lemma 2.8 and (4.4), we get

$$
\left\|U_{0} \cdot \nabla U_{0}\right\|_{L_{T}^{1} \dot{B}_{q, 1}^{3 / q-1}} \leq C\left\|U_{0}\right\|_{\widetilde{L}_{T}^{2} \dot{B}_{p, 1}^{3 / p}}\left\|U_{0}\right\|_{\widetilde{L}_{T}^{2} \dot{B}_{q, 1}^{3 / q}} \leq C \frac{T 2^{N(3 / q-3 / p+2)}}{C(N)^{2}}
$$

and

$\left\|U_{0} \cdot \nabla \widetilde{U}\right\|_{L_{T}^{1} \dot{B}_{q, 1}^{3 / q-1}} \leq C\left\|U_{0}\right\|_{\widetilde{L}_{T}^{2} \dot{B}_{p, 1}^{3 / p}}\|\nabla \widetilde{U}\|_{\widetilde{L}_{T}^{2} \dot{B}_{q, 1}^{3 / q-1}}+C\left\|U_{0}\right\|_{L_{T}^{1} \dot{B}_{p, 1}^{3 / p+1}}\|\nabla \widetilde{U}\|_{\widetilde{L}_{T}^{\infty} \dot{B}_{q, 1}^{3 / q-2}}$

$$
\leq C \frac{T^{1 / 2} 2^{N}+T 2^{2 N}}{C(N)} Y_{T}
$$

Thanks to Lemma 2.6, we have

$$
\begin{aligned}
\|\widetilde{U} \cdot \nabla u\|_{L_{T}^{1} \dot{B}_{q, 1}^{3 / q-1}} & \leq C\|\widetilde{U}\|_{L_{T}^{\infty} \dot{B}_{q, 1}^{3 / q-1}}\left(\left\|\nabla U_{0}\right\|_{L_{T}^{1} \dot{B}_{q, 1}^{3 / q}}+\|\nabla \widetilde{U}\|_{L_{T}^{1} \dot{B}_{q, 1}^{3 / q}}\right) \\
& \leq C Y_{T}\left(\frac{T 2^{N(3 / q-3 / p+2)}}{C(N)}+Y_{T}\right) .
\end{aligned}
$$

Summing up (4.15)-(4.20), we conclude that

$$
\begin{gathered}
Y_{T} \leq \frac{C T\left(1+2^{N\left(\frac{3}{q}-\frac{3}{p}+2\right)} / C(N)\right)}{C(N)}+\frac{C\left(1+T 2^{N\left(\frac{3}{q}-\frac{3}{p}+2\right)}\right)}{C(N)}\left(1+X_{T}\right)^{3} X_{T}+Z_{T} \\
\quad+\frac{C\left(T^{1 / 2} 2^{N}+T 2^{N\left(\frac{3}{q}-\frac{3}{p}+2\right)}\right)}{C(N)} Y_{T}+C\left(1+X_{T}\right)^{3} X_{T} Z_{T}+C X_{T} Y_{T}+C Y_{T}^{2} .
\end{gathered}
$$

Step 3. The estimate of $Z_{T}$

It follows from Proposition 2.4 that

$$
\begin{aligned}
Z_{T} \leq & C\left\{\left\|(\nabla \widetilde{U})^{2}\right\|_{L_{T}^{1} \dot{B}_{q, 1}^{3 / q-2}}+\left\|\nabla U_{0} \nabla \widetilde{U}\right\|_{L_{T}^{1} \dot{B}_{q, 1}^{3 / q-2}}+\|u \cdot \nabla \theta\|_{L_{T}^{1} \dot{B}_{q, 1}^{3 / q-2}}\right. \\
& +\|L(a) \Delta \theta\|_{L_{T}^{1} \dot{B}_{q, 1}^{3 / q-2}}+\|\theta \operatorname{div} u\|_{L_{T}^{1} \dot{B}_{q, 1}^{3 / q-2}}+\left\|L(a)\left(\nabla U_{0}\right)^{2}\right\|_{L_{T}^{1} \dot{B}_{q, 1}^{3 / q-2}} \\
& \left.+\left\|L(a)(\nabla \widetilde{U})^{2}\right\|_{L_{T}^{1} \dot{B}_{q, 1}^{3 / q-2}}+\left\|L(a) \nabla U_{0} \nabla \widetilde{U}\right\|_{L_{T}^{1} \dot{B}_{q, 1}^{3 / q-2}}\right\} .
\end{aligned}
$$

Next we estimate each term on the right hand side of (4.22). Thanks to Lemma 2.6 and (4.4), we get

$$
\begin{aligned}
\left\|(\nabla \widetilde{U})^{2}\right\|_{L_{T}^{1} \dot{B}_{q, 1}^{3 / q-2}} & +\left\|\nabla U_{0} \nabla \widetilde{U}\right\|_{L_{T}^{1} \dot{B}_{q, 1}^{3 / q-2}} \\
& \leq C\|\nabla \widetilde{U}\|_{\widetilde{L}_{T}^{2} \dot{B}_{q, 1}^{3 / q-1}}^{2}+C\left\|\nabla U_{0}\right\|_{L_{T}^{1} \dot{B}_{q, 1}^{3 / q}}\|\nabla \widetilde{U}\|_{L_{T}^{\infty} \dot{B}_{q, 1}^{3 / q-2}} \\
& \leq C Y_{T}^{2}+\frac{C T 2^{N(3 / q-3 / p+2)}}{C(N)} Y_{T} .
\end{aligned}
$$


Due to $3 / p-3 / q+2>0$ and $3 / \widetilde{p}+3 / q-2>0$, we apply (2.1), Lemma $2.8,(4.4)$ and (4.12) to obtain

$$
\begin{aligned}
& \left\|U_{0} \cdot \nabla \theta\right\|_{L_{T}^{1} \dot{B}_{q, 1}^{3 / q-2}} \leq\left\|U_{0}\right\|_{\widetilde{L}_{T}^{2} \dot{B}_{p, 1}^{3 / p}}\|\nabla \theta\|_{\widetilde{L}_{T}^{2} \dot{B}_{q, 1}^{3 / q-2}}+\left\|U_{0}\right\|_{\widetilde{L}_{T}^{2} \dot{B}_{\widetilde{p}, 1}^{3 / \widetilde{p}}}\|\nabla \theta\|_{\widetilde{L}_{T}^{2} \dot{B}_{q, 1}^{3 / q-2}} \\
& \leq C T^{1 / 2}\left(\frac{2^{N}}{C(N)}+\frac{2^{N\left(\frac{3}{\tilde{p}}-\frac{3}{p}+1\right)}}{C(N)}\right)\left(\left\|\Theta_{0}\right\|_{\widetilde{L}_{T}^{2} \dot{B}_{q, 1}^{3 / q-1}}+\left\|\theta_{1}\right\|_{\widetilde{L}_{T}^{2} \dot{B}_{q, 1}^{3 / q-1}}+\left\|\theta_{2}\right\|_{\widetilde{L}_{T}^{2} \dot{B}_{q, 1}^{3 / q-1}}\right) \\
& (4.24) \leq C T^{1 / 2} \frac{2^{N(3 / \widetilde{p}-3 / p+1)}}{C(N)}\left(\frac{1}{C(N)}+\frac{T 2^{N(3 / q-3 / p+2)}}{C(N)}+Z_{T}\right) .
\end{aligned}
$$

On the other hand, we have, by Lemma 2.6,

$$
\begin{aligned}
\|\widetilde{U} \cdot \nabla \theta\|_{L_{T}^{1} \dot{B}_{q, 1}^{3 / q-2}} & \leq C\|\widetilde{U}\|_{\widetilde{L}_{T}^{2} \dot{B}_{q, 1}^{3 / q}}\|\nabla \theta\|_{\widetilde{L}_{T}^{2} \dot{B}_{q, 1}^{3 / q-2}} \\
& \leq C Y_{T}\left(\frac{1}{C(N)}+\frac{T 2^{N(3 / q-3 / p+2)}}{C(N)}+Z_{T}\right),
\end{aligned}
$$

and

$$
\begin{aligned}
\|L(a) \Delta \theta\|_{L_{T}^{1} \dot{B}_{q, 1}^{3 / q-2}} & \leq C\|L(a)\|_{L_{T}^{\infty} \dot{B}_{q, 1}^{3 / q}}\|\Delta \theta\|_{L_{T}^{1} \dot{B}_{q, 1}^{3 / q-2}} \\
& \leq C\left(1+X_{T}\right)^{3} X_{T}\left(\frac{1}{C(N)}+\frac{T 2^{N(3 / q-3 / p+2)}}{C(N)}+Z_{T}\right),
\end{aligned}
$$

and

$$
\begin{aligned}
\|\theta \operatorname{div} \widetilde{U}\|_{L_{T}^{1} \dot{B}_{q, 1}^{3 / q-2}} & \leq C\|\theta\|_{L_{T}^{1} \dot{B}_{q, 1}^{3 / q}}\|\operatorname{div} \widetilde{U}\|_{L_{T}^{\infty} \dot{B}_{q, 1}^{3 / q-2}} \\
& \leq C\left(\frac{1}{C(N)}+\frac{T 2^{N(3 / q-3 / p+2)}}{C(N)}+Z_{T}\right) Y_{T} .
\end{aligned}
$$

Collecting (2.1), (4.4) and (4.12) with Lemma 2.8, we get

$$
\begin{aligned}
\| & \left(\Theta_{0}+\theta_{2}\right) \operatorname{div} U_{0} \|_{L_{T}^{1} \dot{B}_{q, 1}^{3 / q-2}} \\
& \leq C\left\|\operatorname{div} U_{0}\right\|_{L_{T}^{1} \dot{B}_{p, 1}^{3 / p}}\left\|\Theta_{0}+\theta_{2}\right\|_{L_{T}^{\infty} \dot{B}_{q, 1}^{3 / q-2}}+C\left\|\operatorname{div} U_{0}\right\|_{L_{T}^{1} \dot{B}_{\widetilde{p}, 1}^{3 / \widetilde{p}}}\left\|\Theta_{0}+\theta_{2}\right\|_{L_{T}^{\infty} \dot{B}_{q, 1}^{3 / q-2}} \\
& \leq C T\left(\frac{2^{2 N}}{C(N)}+\frac{2^{N(3 / \widetilde{p}-3 / p+2)}}{C(N)}\right)\left(\left\|\Theta_{0}\right\|_{L_{T}^{\infty} \dot{B}_{q, 1}^{3 / q-2}}+\left\|\theta_{2}\right\|_{L_{T}^{\infty} \dot{B}_{q, 1}^{3 / q-2}}\right) \\
(4.28) & \leq C T \frac{2^{N(3 / \widetilde{p}-3 / p+2)}}{C(N)}\left(\frac{1}{C(N)}+Z_{T}\right),
\end{aligned}
$$

and

(4.29) $\left\|\theta_{1} \operatorname{div} U_{0}\right\|_{L_{T}^{1} \dot{B}_{q, 1}^{3 / q-2}}$

$$
\begin{aligned}
& \leq C\left\|\operatorname{div} U_{0}\right\|_{\widetilde{L}_{T}^{2} \dot{B}_{p, 1}^{3 / p-1}}\left\|\theta_{1}\right\|_{\widetilde{L}_{T}^{2} \dot{B}_{q, 1}^{3 / q-1}}+C\left\|\operatorname{div} U_{0}\right\|_{\widetilde{L}_{T}^{2} \dot{B}_{\widetilde{p}, 1}^{3 / \widetilde{p}-1}}\left\|\theta_{1}\right\|_{\widetilde{L}_{T}^{2} \dot{B}_{q, 1}^{3 / q-1}} \\
& \leq C T^{1 / 2}\left(\frac{2^{N}}{C(N)}+\frac{2^{N(3 / \widetilde{p}-3 / p+1)}}{C(N)}\right) \frac{T 2^{N(3 / q-3 / p+2)}}{C(N)^{2}}
\end{aligned}
$$


and

$$
\begin{aligned}
& \text { (4.30) }\left\|L(a)\left(\nabla U_{0}\right)^{2}\right\|_{L_{T}^{1} \dot{B}_{q, 1}^{3 / q-2}} \\
& \leq C\left(\left\|\nabla U_{0}\right\|_{L_{T}^{\infty} \dot{B}_{p, 1}^{3 / p-1}}\left\|L(a) \nabla U_{0}\right\|_{L_{T}^{1} \dot{B}_{q, 1}^{3 / q-1}}+\left\|\nabla U_{0}\right\|_{L_{T}^{1} \dot{B}_{\widetilde{p}, 1}^{\frac{3}{p}-1}}\left\|L(a) \nabla U_{0}\right\|_{L_{T}^{\infty} \dot{B}_{q, 1}^{3 / q-1}}\right) \\
& \leq C\left\|\nabla U_{0}\right\|_{L_{T}^{1} \dot{B}_{\widetilde{p}, 1}^{3 / \widetilde{p}-1}}\left(\left\|\nabla U_{0}\right\|_{L_{T}^{\infty} \dot{B}_{p, 1}^{3 / p}\|L(a)\|_{L_{T}^{\infty} \dot{B}_{q, 1}^{3 / q-1}}}\right. \\
& \quad+\left\|\nabla U_{0}\right\|_{\left.L_{T}^{\infty} \dot{B}_{\widetilde{p}, 1}^{3 / \widetilde{p}}\|L(a)\|_{L_{T}^{\infty} \dot{B}_{q, 1}^{3 / q-1}}\right)} \\
& \leq C \frac{T 2^{N(6 / \widetilde{p}-6 / p+3)}}{C(N)^{2}}\left(1+X_{T}\right)^{3}\|a\|_{L_{T}^{\infty} \dot{B}_{q, 1}^{3 / q-1}} .
\end{aligned}
$$

By Lemma 2.6 and (4.4), we get

$$
\left\|L(a) \nabla U_{0} \nabla \widetilde{U}\right\|_{L_{T}^{1} \dot{B}_{q, 1}^{3 / q-2}}+\left\|L(a)(\nabla \widetilde{U})^{2}\right\|_{L_{T}^{1} \dot{B}_{q, 1}^{3 / q-2}}
$$

$$
\begin{aligned}
& \leq C\|L(a)\|_{L_{T}^{\infty} \dot{B}_{q, 1}^{3 / q}}\left(\left\|\nabla U_{0} \nabla \widetilde{U}\right\|_{L_{T}^{1} \dot{B}_{q, 1}^{3 / q-2}}+\left\|(\nabla \widetilde{U})^{2}\right\|_{L_{T}^{1} \dot{B}_{q, 1}^{3 / q-2}}\right) \\
& \leq C\|a\|_{L_{T}^{\infty} \dot{B}_{q, 1}^{3 / q}}\left(\left\|\nabla U_{0}\right\|_{L_{T}^{1} \dot{B}_{q, 1}^{3 / q}}\|\nabla \widetilde{U}\|_{L_{T}^{\infty} \dot{B}_{q, 1}^{3 / q-2}}+\|\nabla \widetilde{U}\|_{L_{T}^{1} \dot{B}_{q, 1}^{3 / q}}\|\nabla \widetilde{U}\|_{L_{T}^{\infty} \dot{B}_{q, 1}^{3 / q-2}}\right) \\
& \leq C X_{T}\left(\frac{T 2^{N\left(\frac{3}{q}-\frac{3}{p}+2\right)}}{C(N)} Y_{T}+Y_{T}^{2}\right) .
\end{aligned}
$$

Summing up (4.22)-(4.31), we deduce that

$$
\begin{aligned}
Z_{T} \leq & \frac{C\left(T^{1 / 2} 2^{N(3 / \widetilde{p}-3 / p+1)}+T 2^{N(3 / \widetilde{p}-3 / p+2)}+T^{3 / 2} 2^{N(3 / \widetilde{p}+3 / q-6 / p+3)}\right)}{C(N)^{2}} \\
& +C\left(Y_{T}+\frac{C\left(T^{1 / 2} 2^{N\left(\frac{3}{\tilde{p}}-\frac{3}{p}+1\right)}+T 2^{N\left(\frac{3}{\tilde{p}}-\frac{3}{p}+2\right)}\right)}{C(N)}\right) Z_{T}+C Y_{T}^{2}+C X_{T} Y_{T}^{2} \\
& +C\left(1+X_{T}\right)^{3}\left(Z_{T}+\frac{1+T 2^{N\left(\frac{3}{q}-\frac{3}{p}+2\right)}}{C(N)}+Y_{T} \frac{T 2^{N\left(\frac{3}{q}-\frac{3}{p}+2\right)}}{C(N)}\right)\left(X_{T}+Y_{T}\right) \\
& +\frac{C T 2^{N(6 / \widetilde{p}-6 / p+3)}}{C(N)^{2}}\left(1+X_{T}\right)^{3}\|a\|_{L_{T}^{\infty} \dot{B}_{q, 1}^{3 / q-1} .}
\end{aligned}
$$

Step 4. The completion of the proof.

Due to $\max \{2 / \widetilde{p}-1 / q-1 / p, 3 / 5(1 / q-1 / p)\}<\epsilon$, we can take $N$ big enough such that

$$
\frac{T^{1 / 2} 2^{N(3 / \widetilde{p}-3 / p+1)}}{C(N)} \ll 1, \quad \frac{T 2^{N(3 / q-3 / p+2)}}{C(N)} \ll 1
$$

for any $T \leq T_{0}=2^{-2 N(1+\epsilon)}$. 
Then by a continuous argument, we infer from (4.13), (4.14), (4.21) and (4.32) that for any $T \leq T_{0}$,

$$
\begin{aligned}
& X_{T} \leq \frac{C_{1}\left(1+T 2^{N(3 / q-3 / p+2)}\right)}{C(N)}, \quad Y_{T} \leq \frac{C_{2}\left(1+T 2^{N(3 / q-3 / p+2)}\right)}{C(N)} \\
& Z_{T} \leq \frac{C_{3}\left(1+T^{1 / 2} 2^{N(3 / \widetilde{p}-3 / p+1)}+T 2^{N(3 / q-3 / p+2)}\right)}{C(N)}
\end{aligned}
$$

where the constants $C_{1}, C_{2}, C_{3}$ are independent of $N$. Then we deduce from (4.11) and (4.3) that

$$
\begin{aligned}
\left\|\theta\left(T_{0}\right)\right\|_{\dot{B}_{p, 1}^{3 / p-2}} \geq & \left\|\theta_{1}\left(T_{0}\right)\right\|_{\dot{B}_{p, 1}^{3 / p-2}}-\left\|\Theta_{0}\left(T_{0}\right)\right\|_{\dot{B}_{p, 1}^{3 / p-2}}-\left\|\theta_{2}\left(T_{0}\right)\right\|_{\dot{B}_{p, 1}^{3 / p-2}} \\
\geq & c 2^{N(2-3 / q-3 / p-3 \epsilon)}-C 2^{-\frac{N}{2}(3 / q-3 / p+\epsilon)}-C 2^{N(3 /(2 q)-3 /(2 p)-5 \epsilon / 2)} \\
& -C 2^{N(3 / \widetilde{p}-3 /(2 q)-3 /(2 p)-3 \epsilon / 2)} \\
\geq & \frac{c}{2} 2^{N(2-3 / q-3 / p-3 \epsilon)} .
\end{aligned}
$$

This completes the proof of Theorem 1.2.

\section{References}

[1] Bahouri, H., Chemin, J.-Y. And Danchin, R.: Fourier analysis and nonlinear partial differential equations. Grundlehren der Mathematischen Wissenschaften 343, Springer, Heidelberg, 2011.

[2] Bourgain, J. And Pavlović, N.: Ill-posedness of the Navier-Stokes equations in a critical space in 3D. J. Func. Anal. 255 (2008), no. 9, 2233-2247.

[3] Charve, F. And Danchin, R.: A global existence result for the compressible Navier-Stokes equations in the critical $L^{p}$ framework. Arch. Ration. Mech. Anal. 198 (2010), no. 1, 233-271.

[4] Chen, Q., Miao, C. And Zhang, Z.: Well-posedness in critical spaces for the compressible Navier-Stokes equations with density dependent viscosities. Rev. Mat. Iberoam. 26 (2010), no.3, 915-946.

[5] Chen, Q., Miao, C. And Zhang, Z.: Global well-posedness for compressible Navier-Stokes equations with highly oscillating initial velocity. Comm. Pure Appl. Math. 63 (2010), no. 9, 1173-1224.

[6] Danchin, R.: Global existence in critical spaces for compressible Navier-Stokes equations. Invent. Math. 141 (2000), no. 3, 579-614.

[7] Danchin, R.: Global existence in critical spaces for flows of compressible viscous and heat-conductive gases. Arch. Ration. Mech. Anal. 160 (2001), no. 1, 1-39.

[8] Danchin, R.: Local theory in critical spaces for compressible viscous and heat-conductive gases. Comm. Partial Differential Equations 26 (2001), no. 7-8, $1183-1233$

[9] Danchin, R.: Well-posedness in critical spaces for barotropic viscous fluids with truly not constant density. Comm. Partial Differential Equations 32 (2007), no. 9, 1373-1397. 
[10] Feireisl, E.: Dynamics of viscous vompressible fluids. Oxford Lecture Series in Mathematics and its Applications, Oxford University Press, Oxford, 2004.

[11] Fujita, H. And Kato, T.: On the Navier-Stokes initial value problem. I. Arch. Rational Mech. Anal. 16 (1964), 269-315.

[12] Germain, P.: The second iterate for the Navier-Stokes equation. J. Func. Anal. 255 (2008), no. 9, 2248-2264.

[13] Lions, P.-L: Mathematical topics in fluid mechanics. Vol. 2. Compressible models. Oxford Lecture Series in Mathematics and its Applications 10, Oxford Science Publications, The Clarendon Press, Oxford University Press, New York, 1998.

[14] Matsumura, A. And Nishida, N.: The initial value problem for the equations of motion of compressible viscous and heat-conductive fluids. Proc. Japan Acad. Ser. A Math. Sci. 55 (1979), no. 9, 337-342.

[15] NASH, J.: Le problème de Cauchy pour les équations différentielles d'un fluide général. Bull. Soc. Math. France 90 (1962), 487-497.

[16] Sun, Y., Wang, C. And Zhang, Z.: A Beale-Kato-Majda blow-up criterion for the 3-D compressible Navier-Stokes equations. J. Math. Pures Appl. 95 (2011), no. 1, $36-47$

[17] Sun, Y., Wang, C. And Zhang, Z.: A Beale-Kato-Majda criterion for three dimensional compressible viscous heat-conductive flows. Arch. Ration. Mech. Anal. 201 (2011), no. 2, 727-742.

[18] Xin, Z.: Blowup of smooth solutions to the compressible Navier-Stokes equation with compact density. Comm. Pure Appl. Math. 51 (1998), no. 3, 229-240.

Received January 12, 2014; revised November 12, 2014.

Qionglei Chen: Institute of Applied Physics and Computational Mathematics, P.O. Box 8009, Beijing 100088, P. R. China.

E-mail: chen_qionglei@iapcm.ac.cn

Changxing Miao: Institute of Applied Physics and Computational Mathematics, P.O. Box 8009, Beijing 100088, P. R. China.

E-mail: miao_changxing@iapcm.ac.cn, miao_changxing@aliyun.com

Zhifei Zhang: School of Mathematical Sciences, Peking University, 100871, P.R. China.

E-mail: zfzhang@math.pku.edu.cn

Q. Chen and C. Miao are partly supported by the NSF of China under grants 11171033 , 11171034, and 11231006. C. Miao is also supported by Beijing Center of Mathematics and Information Interdisciplinary Science. Z. Zhang is partly supported by NSF of China under Grant 11371037, Program for New Century Excellent Talents in University. 